\title{
Hot electron dynamics in graphene
}

\author{
by
}

Meng-Chieh Ling

A thesis submitted to the graduate faculty

in partial fulfillment of the requirements for the degree of MASTER OF SCIENCE

\author{
Major: Condensed Matter Physics \\ Program of Study Committee: \\ Joerg Schmalian, Major Professor \\ Jigang Wang \\ Kirill Tuchin \\ Golden Miller
}

Iowa State University

Ames, Iowa

2011

Copyright (c) Meng-Chieh Ling, 2011. All rights reserved. 


\section{All rights reserved}

\section{INFORMATION TO ALL USERS}

The quality of this reproduction is dependent on the quality of the copy submitted.

In the unlikely event that the author did not send a complete manuscript and there are missing pages, these will be noted. Also, if material had to be removed, a note will indicate the deletion.

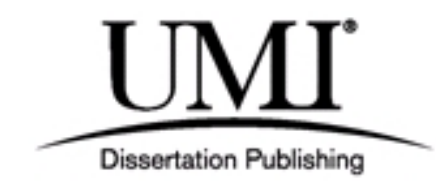

UMI 1494695

Copyright 2011 by ProQuest LLC.

All rights reserved. This edition of the work is protected against unauthorized copying under Title 17, United States Code.

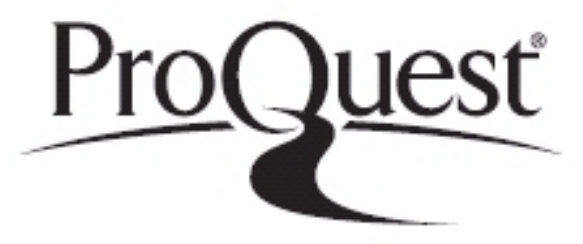

ProQuest LLC.

789 East Eisenhower Parkway

P.O. Box 1346

Ann Arbor, MI 48106 - 1346 


\section{DEDICATION}

I would like to dedicate this thesis to my advisor Joerg Schmailian, a great physicist and mentor. I've learned a lot from him, no matter in physics knowledge or in attitude of doing physics. I also have to thank my fiancee Lena and my family, without their support I would not have been able to complete this work. I would also like to thanks all my friends in this country who help me join the life in a place half the earth away from my hometown, Taiwan. 


\section{TABLE OF CONTENTS}

LIST OF TABLES $\ldots \ldots \ldots \ldots \ldots$

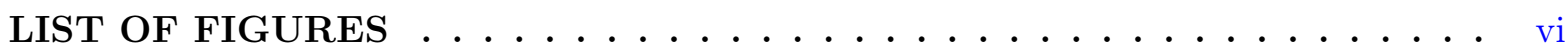

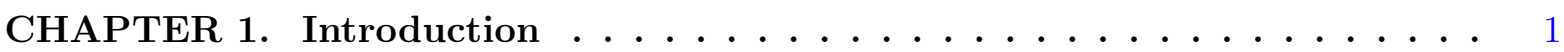

CHAPTER 2. Electronic properties of graphene ............ 5

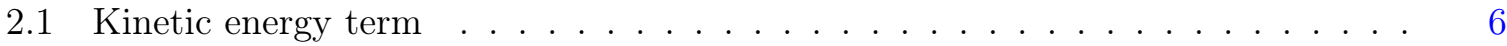

2.2 Current operator for Dirac field in graphene . . . . . . . . . . . . . 10

CHAPTER 3. Summary of theoretical concepts . . . . . . . . . . . 14

3.1 Summary of Boltzmann transport . . . . . . . . . . . . . . . . 14

3.1.1 Derivation of Boltzmann equation . . . . . . . . . . . . . 14

3.1.2 Joule heat created by hot electrons . . . . . . . . . . . . 15

3.1.3 Summary of Arai's method . . . . . . . . . . . . . 17

3.2 The Schwinger mechanism of pair production . . . . . . . . . . . 18

3.3 Electron-phonon coupling in graphene . . . . . . . . . . . . . . 23

3.3 .1 The free phonon part . . . . . . . . . . . . . . 23

3.3.2 Electron-phonon interaction . . . . . . . . . . . . 23

3.3.3 Collision operator due to electron-phonon coupling . . . . . . . . . 26

3.4 Solution of the linearized Boltzmann equation _ . . . . . . . . . . . 26

3.4.1 Coupling to longitudinal accustic phonons . . . . . . . . . . . . 27

3.4.2 Relaxation rate . . . . . . . . . . . . . . . . . . . . 29

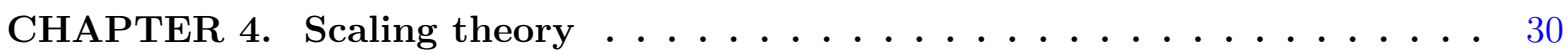

4.1 Trial ansatz . . . . . . . . . . . . . . . . . . . . . . 31

4.1 .1 General dispersion . . . . . . . . . . . . . . . . . . 34 
4.1 .2 Energy density . . . . . . . . . . . . . . . . 35

CHAPTER 5. Relaxation time approximation . . . . . . . . . 37

5.1 Explicit Result for Relaxation Time Approximation . . . . . . . . . . . . . 37

5.2 Scaling argument for relaxation time approximation . . . . . . . . . . . 40

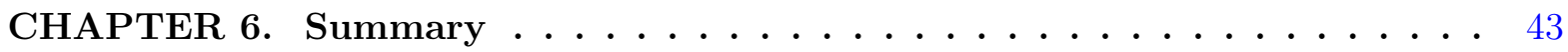

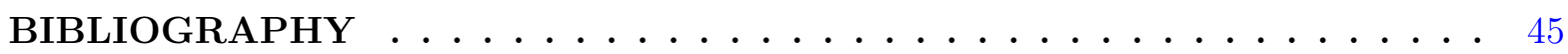




\section{LIST OF TABLES}

Table 3.1 Symbols and their corresponding physical meanings in a phonon scattering potential $U_{S}=D_{A} \frac{\partial u}{\partial x}=K_{\mathbf{q}} A_{\mathbf{q}} e^{ \pm i(\mathbf{q} \cdot \mathbf{x}-\omega t)} \ldots \ldots \ldots$. . . . 


\section{LIST OF FIGURES}

Figure 1.1 Heat transport in graphene attached on a semiconductor substrate. The red lines represent thermal current flowing from high temperature sample to low temperature reservoir. . . . . . . . . . . . . . . .

Figure 1.2 Phonon dispersion relation in graphene. Here we can see that the minimum excitation energy for optical phonon is about $70 \mathrm{meV}$, which corresponds to $840 \mathrm{~K}$. So optical phonon does not contribute to thermal phonon current at low temperature. . . . . . . . . . . .

Figure 2.1 (Color online) Plot of honeycomb crystal lattice of graphene, showing two interpenetrating (red and blue) sub- lattices. . . . . . . . . . . 9

Figure 2.2 Dispersion relation of graphene. . . . . . . . . . . . . . . . . . . . . $\quad 9$

Figure 3.1 Schematic description of temporal evolution of Landau-Zener dynamics. For any arbitrary point in $p$-space, the energy difference between the corresponding two points in the upper and lower band is $\Delta E=2 p v_{F}$. As the time evolves, their energies are getting closer and closer, and reaching the closest distance $\Delta E_{\min }=2 p_{y} v_{F}$ at $t^{*}=\frac{p_{x}}{e E}$; then they move away from each other. The nonadiabatic process induces a transition between the two bands until the transition is completed when the two states are far from each other. In our case we choose at, all the electrons lie in the lower band. . . . . . . . . . . . . . . . . . 


\section{CHAPTER 1. Introduction}

Graphene, a two-dimensional (2D) honeycomb structure allotrope of carbon atoms, has a long history since the invention of the pencil [Petroski (1989)] and the linear dispersion band structure proposed by Wallace [Wal]; however, only after Novoselov et al. successively isolated graphene from graphite [Novoselov et al. (2004)], it has been studied intensively during the recent years. It draws so much attentions not only because of its potential application in future electronic devices but also because of its fundamental properties: its quasiparticles are governed by the two-dimensional Dirac equation, and exhibit a variety of phenomena such as the anomalous integer quantum Hall effect (IQHE) [Novoselov et al. (2005)] measured experimentally, a minimal conductivity at vanishing carrier concentration [Neto et al. (2009)], Kondo effect with magnetic element doping [Hentschel and Guinea (2007)], Klein tunneling in $p$-n junctions [Cheianov and Fal'ko (2006), Beenakker (2008)], Zitterbewegung [Katsnelson (2006)], and Schwinger pair production [Schwinger (1951); Dora and Moessner (2010)]. Although both electron-phonon coupling and photoconductivity in graphene also draws great attention [Yan et al. (2007); Satou et al. (2008); Hwang and Sarma (2008); Vasko and Ryzhii (2008); Mishchenko (2009)], the nonequilibrium behavior based on the combination of electronphonon coupling and Schwinger pair production is an intrinsic graphene property that has not been investigated.

Our motivation for studying clean graphene at low temperature is based on the following effect: for a fixed electric field, below a sufficiently low temperature linear eletric transport breaks down and nonlinear transport dominates. The criteria of the strength of this field [Fritz et al. (2008)] is

$$
e E=T^{2} / \hbar v_{F}
$$

For $T>\sqrt{e E \hbar v_{F}}$ the system is in linear transport regime while for $T<\sqrt{e E \hbar v_{F}}$ the system 
is in nonlinear transport regime. From the scaling's point of view, at the nonlinear transport regime the temperature $T$ and electric field $E$ are also related. In this thesis we show that the nontrivial electron distribution function can be associated with an effective temperature $T^{*}$ which exhibits a dependence on electric field $E$ and electron-phonon coupling $g$ :

$$
T^{*} \propto \frac{E^{1 / 4}}{g}
$$

The anamolous exponent $\frac{1}{4}$ may obtained from scaling. Meanwhile, yet we cannot obtain the distribution function, however, argument based on scaling gives us the current dependence on electric field:

$$
J \propto \frac{\sqrt{E}}{g^{2}}
$$

which is a very different result compared with the results in which electrons do not experience scattering. This result provides us with important insighht into the correct nonequilibrium distribution function because now we know what the electric field dependence of current must be.

Due to the applied field, the electronic system produces heat which prevents us from reaching a steady state. In order to remove Joule heat, we imagine that we have a graphene flake attached to a semiconductor substrate. Joule heat either transport to its environment or to the substrate as shown in 1.1. The red lines represent heat current flowing from high temperature sample to the low temperature reservoir. However, for a very large system, the temperature gradient is 0 in the plane so heat cannot be conducted outside in the horizontal direction, while the energy gap in semiconductor also forbids electron current from flowing into the substrate. But for phonon thermal current, the temperature gradient is large in the vertical direction, so heat can be transported into the substrate via phonons. There are two possible channels of phonon degrees of freedom, acoustic phonon and optical phonon. As we can see from Fig. 1.2 [Kusminskiy et al. (2009)], since the optical phonon excitation energy is too large for a low temperature system, it is note likely to be excited by the nonlinear electric field, so the possible way left is by electron-acoustic phonon scattering. Here acoustic phonon acts as a heat bath to absorb the Joule heat created by pair production process. Hence the scattering process is determined by electron-acoustic phonon interaction which will be introduced in section 3.3. 

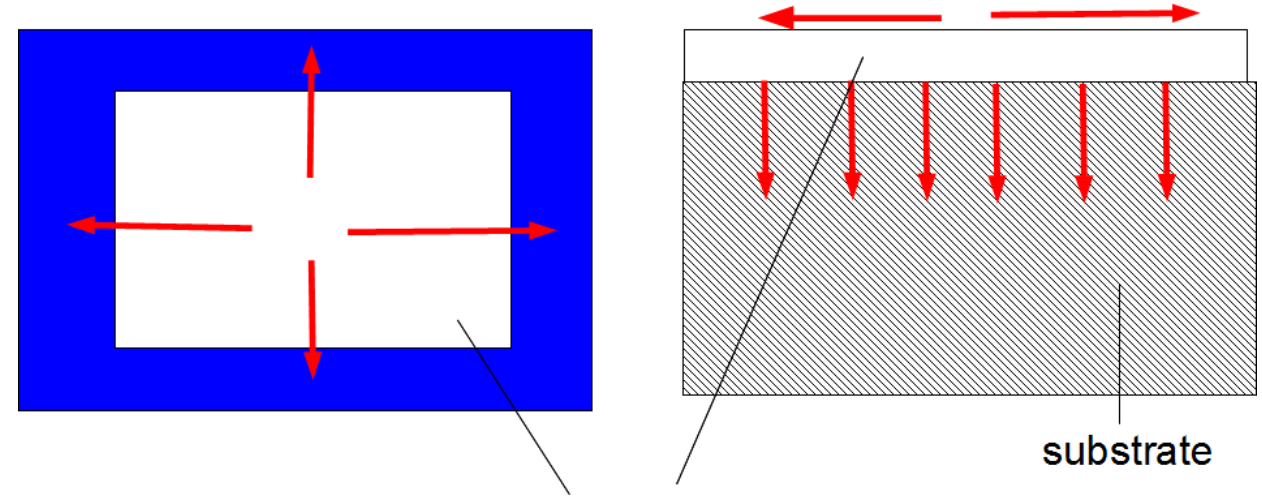

\section{Graphene}

Figure 1.1 Heat transport in graphene attached on a semiconductor substrate. The red lines represent thermal current flowing from high temperature sample to low temperature reservoir.

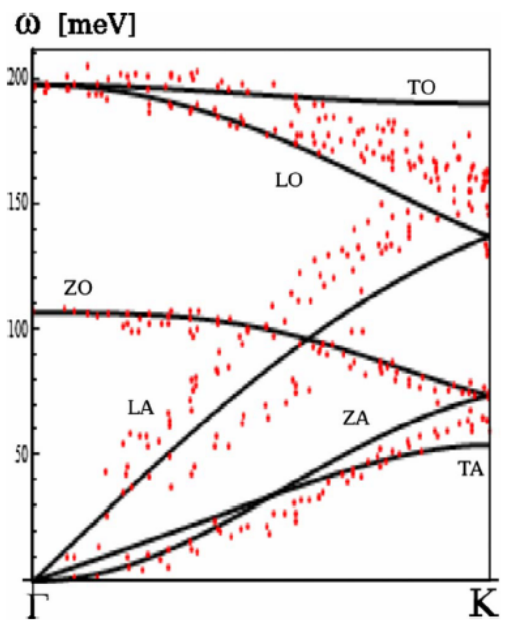

Figure 1.2 Phonon dispersion relation in graphene. Here we can see that the minimum excitation energy for optical phonon is about $70 \mathrm{meV}$, which corresponds to $840 \mathrm{~K}$. So optical phonon does not contribute to thermal phonon current at low temperature. 
This thesis is organized as follows: electronic properties such as linear band structure, current for Dirac fermions, and electron-phonon coupling will be introduced in chapter 2; theoretical concepts like Boltzmann equation with electron-phonon coupling, Schwinger mechanism, and effective temperature will be included in chapter 3 ; we will use scaling to obtain the electric current as well as the relation between effective temperature $T^{*}$, electric field, and electronphonon couppling constant in nonlinear regime in chapter 4 . In the end, we will show that in relaxation time approximation, both explicitly solving distribution function and scaling in relaxation time approximation give us the same result which confirms the advantage of using scaling as a tool to obtain the correct nonlinear transport behavior. 


\section{CHAPTER 2. Electronic properties of graphene}

At low energies, graphene can be described by a theory of $\mathrm{N}=4$ two-component fermions. The two components correspond to fermions associated with the two sublattices of the honeycomb lattice structure, and $\mathrm{N}=4$ corresponds to 2 symmetry inequivalent nodes of the dispersion and 2 for spin. To obtain these results, it is sufficient to start from a tight-binding model with Hamiltonian $H=H_{0}$ of spinless fermions $c_{i}$ hopping on the honeycomb lattice. The kinetic energy is

$$
H_{0}=-t \sum_{i, j} c_{i}^{\dagger} c_{j}
$$

The honeycomb lattice (2.1) of nearest-neighbor distance a consists of two interpenetrating triangular lattices with lattice spacing $\sqrt{3 a}$. We define blue (B) and red (R) sublattices with the fermions on the respective sublattices given by

$$
c_{i}= \begin{cases}a_{i} & \text { for } i \in R \\ b_{i} & \text { for } i \in B\end{cases}
$$

The lattice vectors for the $R$ and $B$ lattices are (upper and lower corresponding to x and $\mathrm{y}$, respectively):

$$
\begin{gathered}
\mathbf{s}_{1}=\sqrt{3} a\left(\begin{array}{c}
1 \\
0
\end{array}\right) \text { and } \mathbf{s}_{2}=\sqrt{3} a\left(\begin{array}{c}
\frac{1}{2} \\
-\frac{\sqrt{3}}{2}
\end{array}\right) \text {, R sublattice } \\
\mathbf{s}_{1}=\sqrt{3} a\left(\begin{array}{c}
1 \\
0
\end{array}\right) \text { and } \mathbf{s}_{2}=\sqrt{3} a\left(\begin{array}{c}
\frac{1}{2} \\
\frac{\sqrt{3}}{2}
\end{array}\right) \text {, B sublattice }
\end{gathered}
$$

The reciprocal lattice vectors are 


$$
\begin{gathered}
\mathbf{b}_{1}=\frac{4 \pi}{3 a}\left(\begin{array}{c}
\frac{\sqrt{3}}{2} \\
\frac{1}{2}
\end{array}\right) \text { and } \mathbf{b}_{2}=\frac{4 \pi}{3}\left(\begin{array}{c}
0 \\
-1
\end{array}\right), \text { R sublattice } \\
\mathbf{b}_{1}=\frac{4 \pi}{3 a}\left(\begin{array}{c}
\frac{\sqrt{3}}{2} \\
-\frac{1}{2}
\end{array}\right) \text { and } \mathbf{b}_{2}=\frac{4 \pi}{3}\left(\begin{array}{c}
0 \\
1
\end{array}\right), \text { B sublattice }
\end{gathered}
$$

We also need to define the vectors $\mathbf{u}$ and $\mathbf{v}$ that connect the two sublattices. The three vectors that point from the $\mathrm{R}$ sublattice to the $\mathrm{B}$ sublattice are

$$
\begin{aligned}
& \mathbf{u}_{1}=-a \hat{y} \\
& \mathbf{u}_{2}=a\left(\frac{\sqrt{3}}{2} \hat{x}+\frac{1}{2} \hat{y}\right) \\
& \mathbf{u}_{3}=a\left(-\frac{\sqrt{3}}{2} \hat{x}+\frac{1}{2} \hat{y}\right)
\end{aligned}
$$

while the vectors $\mathbf{v}_{i}$ that point from the Blue sublattice to the red sublattice are clearly the minus of the $\mathbf{u}_{i}$ :

$$
\begin{aligned}
& \mathbf{v}_{1}=a \hat{y} \\
& \mathbf{v}_{2}=-a\left(\frac{\sqrt{3}}{2} \hat{x}+\frac{1}{2} \hat{y}\right) \\
& \mathbf{v}_{3}=a\left(\frac{\sqrt{3}}{2} \hat{x}-\frac{1}{2} \hat{y}\right)
\end{aligned}
$$

Next, we derive a Dirac-like Hamiltonian for $H$, starting with the analysis of $H_{0}$.

\subsection{Kinetic energy term}

In tight binding model the kinetic energy of electrons in grapene can be written as

$$
H_{0}=-t\left[\sum_{i \in R, j \mathrm{n}, \mathrm{n}} a_{i}^{\dagger} b_{j}+\sum_{i \in B, j \mathrm{n}, \mathrm{n}} b_{i}^{\dagger} a_{j}\right]
$$

now we do Fourier transformation to transform the creation and annihilation operators from real space to momentum space, such as

$$
b_{i}=\sum_{\mathbf{q}} e^{-i \mathbf{q} \cdot \mathbf{r}_{i}} b_{q}
$$


thus

$$
\begin{aligned}
H_{0} & =-t\left(\sum_{i \in R, j} \sum_{\mathrm{n}, \mathrm{n}} e_{\mathbf{k}, \mathbf{q}}^{i\left(\mathbf{k} \cdot \mathbf{r}_{i}-\mathbf{q} \cdot \mathbf{r}_{j}\right)} a_{k}^{\dagger} b_{q}+\sum_{i \in B, j} \sum_{\mathrm{n}, \mathrm{n}} e_{\mathbf{k}, \mathbf{q}}^{i\left(\mathbf{k} \cdot \mathbf{r}_{i}-\mathbf{q} \cdot \mathbf{r}_{j}\right)} b_{k}^{\dagger} a_{q}\right) \\
& =-t\left(\sum_{i \in R} \sum_{\mathbf{u}_{i}} \sum_{\mathbf{k}, \mathbf{q}} e^{i(\mathbf{k}-\mathbf{q}) \cdot \mathbf{r}_{i}-i \mathbf{q} \cdot \mathbf{u}_{i}} a_{k}^{\dagger} b_{q}+\sum_{i \in B} \sum_{\mathbf{v}_{i}} \sum_{\mathbf{k}, \mathbf{q}} e^{i(\mathbf{k}-\mathbf{q}) \cdot \mathbf{r}_{i}-i \mathbf{q} \cdot \mathbf{v}_{i}} b_{k}^{\dagger} a_{q}\right)
\end{aligned}
$$

where the summation over $\mathbf{u}_{i}$ and $\mathbf{v}_{i}$ gives us

$$
\begin{aligned}
c_{u}(\mathbf{q}) & \equiv \sum_{\mathbf{u}_{i}} e^{-i \mathbf{q} \cdot \mathbf{u}_{i}} \\
& =e^{i q_{y} a}+2 \cos \left[\frac{\sqrt{3}}{2} q_{x} a\right] e^{-\frac{i}{2} q_{y} a}
\end{aligned}
$$

and

$$
\begin{aligned}
c_{v}(\mathbf{q}) & \equiv \sum_{\mathbf{v}_{i}} e^{-i \mathbf{q} \cdot \mathbf{v}_{i}} \\
& =e^{-i q_{y} a}+2 \cos \left[\frac{\sqrt{3}}{2} q_{x} a\right] e^{\frac{i}{2} q_{y} a}
\end{aligned}
$$

The sums over $\mathbf{r}_{i}$ then yield delta functions constraining $\mathbf{k}=\mathbf{q}$, so that we finally obtain

$$
\begin{aligned}
H_{0} & =-t \sum_{\mathbf{k}}\left(c_{u}(\mathbf{k}) a_{k}^{\dagger} b_{k}+c_{v}(\mathbf{k}) b_{k}^{\dagger} a_{k}\right) \\
& =-t \sum_{\mathbf{k}}\left(\begin{array}{ll}
a_{k}^{\dagger} & b_{k}^{\dagger}
\end{array}\right)\left(\begin{array}{cc}
0 & c_{u}(\mathbf{k}) \\
c_{v}(\mathbf{k}) & 0
\end{array}\right)\left(\begin{array}{l}
a_{k} \\
b_{k}
\end{array}\right)
\end{aligned}
$$

where $\mathbf{k}$ is sitting inside the first Brillioun zone (BZ) and now we can diagonalize the $2 \times 2$ matrix to obtain the spectrum of eigenvalues:

$$
E(\mathbf{k})^{2}=t^{2} c_{u}(\mathbf{k}) c_{v}(\mathbf{k})
$$

or,

$$
E(\boldsymbol{k})= \pm t \sqrt{1+4 \cos ^{2}\left[\frac{\sqrt{3}}{2} k_{x} a\right]+4 \cos \left[\frac{\sqrt{3}}{2} k_{x} a\right] \cos \left[\frac{3}{2} k_{y} a\right]}
$$


The dispersion $E(\boldsymbol{k})$ has nodes at periodic positions in k-space, with the six closest to the origin being at $\frac{4 \pi}{3}\left( \pm \frac{1}{\sqrt{3}}, 0\right)$ and $\frac{4 \pi}{3}\left( \pm \frac{1}{2 \sqrt{3}}, \pm \frac{1}{2}\right)$. Two of these are inequivalent, i.e., not connected by the reciprocal lattice vectors $\mathbf{b}_{i}$. The result is shown in 2.2 .

We construct our Dirac theory by expanding $H_{0}$ near the two nodes

$$
\begin{gathered}
\mathbf{k}_{1}=\frac{4 \pi}{3 a}\left(\frac{1}{\sqrt{3}}, 0\right)=\frac{4 \pi}{3 \sqrt{3} a} \hat{x}, \\
\mathbf{k}_{2}=\frac{4 \pi}{3 a}\left(\frac{1}{2 \sqrt{3}},-\frac{1}{2}\right)=\frac{2 \pi}{3 \sqrt{3} a} \hat{x}-\frac{2 \pi}{3 a} \hat{y} .
\end{gathered}
$$

and we may expand the Hamiltonian near the two nodes ( $K$ and $K^{\prime}$ points):

$$
H_{o} \simeq-t \sum_{\mathbf{k}} \sum_{i=1,2}\left(\begin{array}{ll}
a_{\mathbf{k}+\mathbf{k}_{i}}^{\dagger} & b_{\mathbf{k}+\mathbf{k}_{i}}^{\dagger}
\end{array}\right)\left(\begin{array}{cc}
0 & c_{u}\left(\mathbf{k}+\mathbf{k}_{i}\right) \\
c_{v}\left(\mathbf{k}+\mathbf{k}_{i}\right) & 0
\end{array}\right)\left(\begin{array}{c}
a_{\mathbf{k}+\mathbf{k}_{i}} \\
b_{\mathbf{k}+\mathbf{k}_{i}}
\end{array}\right)
$$

Now the $\mathrm{k}$ points are within the triangles whose centers are the two distinguished $\mathrm{K}$ points respectively with $\Gamma$ points as the apexs. With the $\mathrm{K}$ points fixed and expanding to the first order term, we may obtain:

$$
\begin{aligned}
H_{0} & \simeq-\frac{3}{2} t a \sum_{\mathbf{k}, i} \psi_{i}^{\dagger}(\mathbf{k})\left(\begin{array}{cc}
0 & -k_{x}+i k_{y} \\
-k_{x}-i k_{y} & 0
\end{array}\right) \psi_{i}(\mathbf{k}) \\
& =v \sum_{\mathbf{k}} \sum_{i=1,2} \psi_{i}^{\dagger}(\mathbf{k}) \mathbf{k} \cdot \sigma \psi_{i}(\mathbf{k})
\end{aligned}
$$

where

$$
\begin{aligned}
& \psi_{1}(\mathbf{k})=\left(\begin{array}{c}
a_{\mathbf{k}+\mathbf{k}_{1}} \\
b_{\mathbf{k}+\mathbf{k}_{1}}
\end{array}\right) \\
& \psi_{2}(\mathbf{k})=\left(\begin{array}{c}
b_{\mathbf{k}+\mathbf{k}_{2}} e^{\frac{i \pi}{3}} \\
a_{\mathbf{k}+\mathbf{k}_{2}}
\end{array}\right)
\end{aligned}
$$

This matrix is diagonalized by

$$
U_{\mathbf{k}}=\frac{e^{i \varphi_{\mathbf{k}}}}{\sqrt{2}}\left(\begin{array}{cc}
e^{i\left(\chi_{\mathbf{k}}+\theta_{\mathbf{k}}\right)} & e^{i \chi_{\mathbf{k}}} \\
-e^{-i \chi_{\mathbf{k}}} & e^{-i\left(\chi_{\mathbf{k}}+\theta_{\mathbf{k}}\right)}
\end{array}\right)
$$




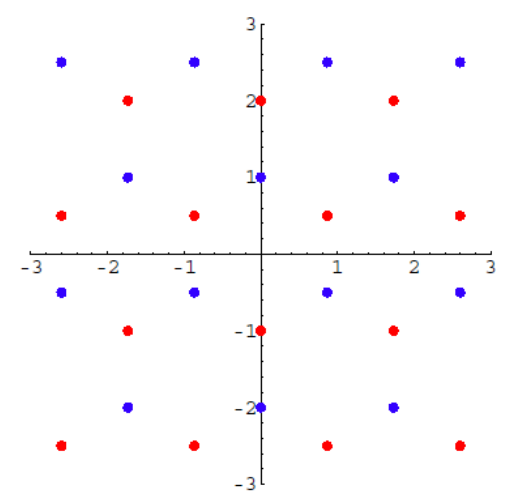

Figure 2.1 (Color online) Plot of honeycomb crystal lattice of graphene, showing two interpenetrating (red and blue) sub- lattices.

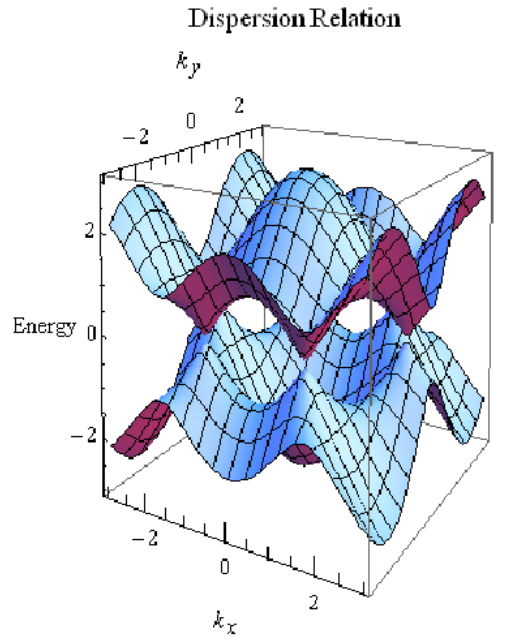

Figure 2.2 Dispersion relation of graphene. 
with arbitrary $\chi_{\mathbf{k}}$ and $\varphi_{\mathbf{k}}$ i.e.

$$
U_{\mathbf{k}} v \hbar \mathbf{k} \cdot \sigma U_{\mathbf{k}}^{-1}=\left(\begin{array}{cc}
v \hbar k & 0 \\
0 & -v \hbar k
\end{array}\right) .
$$

The eigenvalues of the Hamiltonian are $\pm v \hbar k$. Thus we obtain new quasiparticle states for the two bands: $\gamma_{\mathbf{k}}=U_{\mathbf{k}} \psi_{\mathbf{k}}$, with

$$
H_{0, \mathrm{el}}=v \hbar \int_{\mathbf{k}} \sum_{\lambda= \pm} \lambda k \gamma_{\mathbf{k}, \lambda}^{\dagger} \gamma_{\mathbf{k}, \lambda}
$$

The eigenvalues are therefore

$$
\varepsilon_{\mathbf{k} \lambda}=\lambda v \hbar k
$$

A convenient choice is $\chi_{\mathbf{k}}=-\theta_{\mathbf{k}} / 2$ where:

$$
U_{\mathbf{k}}=\frac{e^{i \varphi_{\mathbf{k}}}}{\sqrt{2}}\left(\begin{array}{cc}
e^{i \theta_{\mathbf{k}} / 2} & e^{-i \theta_{\mathbf{k}} / 2} \\
-e^{i \theta_{\mathbf{k}} / 2} & e^{-i \theta_{\mathbf{k}} / 2}
\end{array}\right)
$$

\subsection{Current operator for Dirac field in graphene}

In this section we derive the current operator of Dirac fermion in graphene. We expect to use the continuity equation $\frac{\partial \rho}{\partial t}+\nabla \cdot \mathbf{j}=0$ to find the current in graphene. Since

$$
\begin{aligned}
\rho & =e \bar{\psi} \psi \\
& =e \sum_{\alpha}\left(\psi_{\alpha}^{\dagger} \psi_{\alpha}\right)
\end{aligned}
$$

and we need a relation to connect the time derivatives and space derivatives of creation and annihilation operators, we may think about Heisenberg equation of motion

$$
\frac{d A}{d t}=\frac{1}{i \hbar}[A, H]
$$

In order to get the space derivatives, we Fourier transform the bare Hamiltonian:

$$
\begin{aligned}
H_{0} & =v \hbar \int \frac{d \mathbf{k}}{(2 \pi)^{2}} \bar{\psi}(\mathbf{k}) \mathbf{k} \cdot \boldsymbol{\sigma} \psi(\mathbf{k}) \\
& =-i v \hbar \int d \mathbf{r} \bar{\psi}(\mathbf{r}) \boldsymbol{\sigma} \cdot \nabla \psi(\mathbf{r})
\end{aligned}
$$


Now

$$
\begin{aligned}
\frac{d \psi_{1}}{d t} & =\frac{1}{i \hbar}\left[\psi_{1}, H\right] \\
& =-v\left(\frac{\partial}{\partial x}-i \frac{\partial}{\partial y}\right) \psi_{2}
\end{aligned}
$$

similarly

$$
\begin{aligned}
\frac{d \psi_{2}}{d t} & =\frac{1}{i \hbar}\left[\psi_{2}, H\right] \\
& =-v\left(\frac{\partial}{\partial x}+i \frac{\partial}{\partial y}\right) \psi_{1}
\end{aligned}
$$

what about the electron-phonon interaction contribution? Well, as you calculate $\frac{\partial \rho}{\partial t}$, you will find its contribution to current is 0 .

As we mentioned before $\rho=e\left(\psi_{1}^{\dagger} \psi_{1}+\psi_{2}^{\dagger} \psi_{2}\right)$, so

$$
\begin{aligned}
\frac{\partial \rho}{\partial t}= & e\left(\dot{\psi}_{1}^{\dagger} \psi_{1}+\psi_{1}^{\dagger} \dot{\psi}_{1}+\dot{\psi}_{2}^{\dagger} \psi_{2}+\psi_{2}^{\dagger} \dot{\psi}_{2}\right) \\
= & -e v\left[-\psi_{1}\left(\frac{\partial}{\partial x}+i \frac{\partial}{\partial y}\right) \psi_{2}^{\dagger}+\psi_{1}^{\dagger}\left(\frac{\partial}{\partial x}-i \frac{\partial}{\partial y}\right) \psi_{2}\right. \\
& \left.-\psi_{2}\left(\frac{\partial}{\partial x}-i \frac{\partial}{\partial y}\right) \psi_{1}^{\dagger}+\psi_{2}^{\dagger}\left(\frac{\partial}{\partial x}+i \frac{\partial}{\partial y}\right) \psi_{1}\right]
\end{aligned}
$$

Now we need to find out $\mathbf{j}$ such that

$$
\frac{\partial \rho}{\partial t}=-\left(\frac{\partial j_{x}}{\partial x}+\frac{\partial j_{j}}{\partial y}\right)
$$

then

$$
\begin{aligned}
\frac{\partial j_{x}}{\partial x} & =-e v\left(\psi_{1} \frac{\partial \psi_{2}^{\dagger}}{\partial x}-\psi_{1}^{\dagger} \frac{\partial \psi_{2}}{\partial x}+\psi_{2} \frac{\partial \psi_{1}^{\dagger}}{\partial x}-\psi_{2}^{\dagger} \frac{\partial \psi_{1}}{\partial x}\right) \\
\Rightarrow j_{x} & =e v \bar{\psi} \sigma_{1} \psi
\end{aligned}
$$

similarly,

$$
\begin{aligned}
\frac{\partial j_{y}}{\partial y} & =-i e v\left(\psi_{1} \frac{\partial \psi_{2}^{\dagger}}{\partial y}+\psi_{1}^{\dagger} \frac{\partial \psi_{2}}{\partial y}-\psi_{2} \frac{\partial \psi_{1}^{\dagger}}{\partial y}-\psi_{2}^{\dagger} \frac{\partial \psi_{1}}{\partial y}\right) \\
\Rightarrow j_{y} & =e v \bar{\psi} \sigma_{2} \psi
\end{aligned}
$$

so we have $\mathbf{j}=e v \bar{\psi} \boldsymbol{\sigma} \psi$.

We use the property (valid for $\chi_{\mathbf{k}}=-\theta_{\mathbf{k}} / 2$ ) that

$$
U_{\mathbf{k}} \boldsymbol{\sigma} U_{\mathbf{k}}^{-1}=\frac{\mathbf{k}}{k} \sigma_{z}-\frac{\mathbf{k} \times \mathbf{e}_{z}}{k} \sigma_{y}
$$


and obtain for the current an intra- and inter-band contribution:

$$
\mathbf{j}=\mathbf{j}_{\text {intra }}+\mathbf{j}_{\text {inter }}
$$

with:

$$
\begin{aligned}
& \mathbf{j}_{\text {intra }}=e v N \int_{\mathbf{k}} \sum_{\lambda= \pm} \frac{\lambda \mathbf{k}}{k} \gamma_{\mathbf{k}, \lambda}^{\dagger} \gamma_{\mathbf{k}, \lambda} \\
& \mathbf{j}_{\text {inter }}=\operatorname{ievN} \int_{\mathbf{k}} \frac{\mathbf{k} \times \mathbf{e}_{z}}{k}\left(\gamma_{\mathbf{k},+}^{\dagger} \gamma_{\mathbf{k},-}-\gamma_{\mathbf{k},-}^{\dagger} \gamma_{\mathbf{k},+}\right)
\end{aligned}
$$

In what follows we concentrate our attention to intraband currents. We introduce the distribution function $f_{\mathbf{k} \lambda}(t)$ for the single particle occupation of a state with momentum $\mathbf{k}$ and band index $\lambda$ :

$$
f_{\mathbf{k} \lambda}(t)=\left\langle\gamma_{\mathbf{k}, \lambda}^{\dagger} \gamma_{\mathbf{k}, \lambda}\right\rangle
$$

It follows for the intraband current that

$$
\left\langle\mathbf{j}_{\text {intra }}\right\rangle=e v N \int_{\mathbf{k}} \sum_{\lambda= \pm} \frac{\lambda \mathbf{k}}{k} f_{\mathbf{k} \lambda}(t) .
$$

Within linear response, we make the ansatz

$$
f_{\mathbf{k} \lambda}(t)=f_{0}(\lambda v k)+e \lambda \frac{\mathbf{E} \cdot \mathbf{k}}{k} f_{0}(v k)\left(1-f_{0}(v k)\right) g_{\lambda}(k, t),
$$

where

$$
f_{0}(\varepsilon)=\frac{1}{e^{\beta \omega}+1}
$$

is the distribution funtion in equilibrium, while $g_{\lambda}(k, t)$ parametrizes the electric field induced deviations in the occupation from its equilibrium value. $g_{\lambda}(k, t)$ will be determined from a solution of the Boltzmann equation. Since the current vanishes for $f_{\mathbf{k} \lambda}(t)=f_{0}(\lambda v k)$, it follows

$$
\left\langle\mathbf{j}_{\text {intra }}\right\rangle=e^{2} v N \int_{\mathbf{k}} \frac{\mathbf{k}(\mathbf{E} \cdot \mathbf{k})}{k^{2}} f_{0}(v k)\left(1-f_{0}(v k)\right) \sum_{\lambda= \pm} g_{\lambda}(k, t) .
$$

In case $\langle\mathbf{j}\rangle=\left\langle\mathbf{j}_{\text {intra }}\right\rangle$ follows for the conductivity $\left\langle j_{\alpha}\right\rangle=\sum_{\beta} \sigma_{\alpha \beta} E_{\beta}$ that

$$
\sigma_{\alpha \beta}=e^{2} v N \int_{\mathbf{k}} \frac{k_{\alpha} k_{\beta}}{k^{2}} f_{0}(v k)\left(1-f_{0}(v k)\right) \sum_{\lambda= \pm} g_{\lambda}(k, t)
$$


By symmetry follows $\sigma=\sigma_{x x}=\sigma_{y y}$ such that

$$
\begin{aligned}
\sigma & =\frac{e^{2} v}{2} N \int_{\mathbf{k}} f_{0}(v k)\left(1-f_{0}(v k)\right) \sum_{\lambda= \pm} g_{\lambda}(k, t) \\
& =e^{2} v \pi N \int_{0}^{\infty} d k k f_{0}(v k)\left(1-f_{0}(v k)\right) \sum_{\lambda= \pm} g_{\lambda}(k, t)
\end{aligned}
$$




\section{CHAPTER 3. Summary of theoretical concepts}

To obtain the nonequilibrium distribution function, Boltzmann transport theory, which will be introduced in section 3.1, is used as a powerful tool to study the problem. Not only does it lead us to the equation for distribution function, but also enable us using scaling to investigate the nonlinear transport properties. Furthermore, we will also illusrate how biased electric field produces heat in electronic system by elastic scattering (like impurity) in section 3.1. In section 3.2 , it will be shown how to use Dirac equation with an electric field, which acts as shifting the Dirac point to $(e E t, 0)$ and resulting in pair-production, to exhibit linear transport at low electric field while gives us nonlinear transport at high electric field. The resulting Hamiltonian shows the same structure as Landau-Zener model [Zener (1932)] therefore we can always obtain the distribution function in the long time limit. Also, the currents due to Kubo mechanism and Schwinger/Kibble-Zurek mechanism may also be obtained at large field limit. In secion 3.3, we will explain the idea of effective temperature and shows the electric field temperature dependence under the relaxation time approximation in which both elastic and inelastic scattering are constants.

\subsection{Summary of Boltzmann transport}

\subsubsection{Derivation of Boltzmann equation}

In this section, we use semiclassical argument to qualitatively illustrate how to we build up Boltzmann equation which is used to study the transport process [Grosso and Parravicini (2000)]. We all know that at thermodynamic equilibrium, the distribution function $f_{0}$ obeys the Fermi-Dirac (F-D) distribution function

$$
f_{0}(\mathbf{k})=\frac{1}{e^{(E(\mathbf{k})-\mu) / k_{B} T}+1} .
$$


where $T$ is the temperature of the system and $\mu$ is the chemical potential. However, life is not this easy in non-equilibrium state. When external perturbations (electric fields, magnetic fields, temperature gradients) are applied to the system, the distribution function no longer obeys the F-D distribution function. In general the disturbed distribution function $f(\mathbf{r}, \mathbf{k}, t)$, in additio to $\mathbf{k}$, depends also on the real space coordinate $\mathbf{r}$, and on time $t$.

According to the semiclassical dynamics of carriers with energy $E(\mathbf{k})$ and momentum $\mathbf{k}$ in given energy band has velocity

$$
\mathbf{v}_{\mathbf{k}}=\frac{1}{\hbar} \frac{\partial E}{\partial \mathbf{k}}
$$

and experience force

$$
\mathbf{F}=\frac{d(\hbar \mathbf{k})}{d t}
$$

During the motion, collision processes may cause a net rate of change $\left[\frac{\partial f}{\partial t}\right]_{\text {coll }}$, which is determined by the interaction, of the number of electron in the phase space volume $d \mathbf{r} d \mathbf{k}$. Using Liouville theorem (volumes in phase space are preserved by the semiclassical equation of motion) we must have for the distribution function

$$
f(\mathbf{r}+d \mathbf{r}, \mathbf{k}+d \mathbf{k}, t+d t) \equiv f(\mathbf{r}, \mathbf{k}, t)+\left[\frac{\partial f}{\partial t}\right]_{\text {coll }} d t
$$

Expanding 3.1 in Taylor series up to the first order, and we obtain the Boltzmann equation

$$
\frac{\partial f}{\partial \mathbf{r}} \cdot \mathbf{v}+\frac{\mathbf{F}}{\hbar} \cdot \frac{\partial f}{\partial \mathbf{k}}+\frac{\partial f}{\partial t}=\left[\frac{\partial f}{\partial t}\right]_{\text {coll }}
$$

In our case, the $\mathbf{F}$ is the eletric force $e \mathbf{E}$ and $\left[\frac{\partial f}{\partial t}\right]_{\text {coll }}$ is determined by eletron-phonon interaction which will be discussed in detail in section 3.3.

\subsubsection{Joule heat created by hot electrons}

To qualitatively illustrate how non-equilibrium state generates Joule heat, we introduce the relaxation times approximation by requiring $\left[\frac{\partial f}{\partial t}\right]_{\text {coll }}=-\frac{f-\bar{f}}{\tau}$ in 3.1 with elastic impurity scattering rate $\tau^{-1}$ characterizs the relaxation of the electronic distribution function $f(\mathbf{p})$ to its angular average $\bar{f}(p)$. So we obtain

$$
\frac{\partial f}{\partial t}+e \mathbf{E} \cdot \nabla_{p} f+\mathbf{v} \cdot \nabla_{x} f=-\frac{f-\bar{f}}{\tau}
$$


Here we set $\hbar=1$. Assuming the solution can be expanded in powers of the applied field, we write

$$
f=f^{(0)}+f^{(1)}+f^{(2)}+\ldots
$$

with the assumptions that

$$
f^{(n)} \ll f^{(n-1)},
$$

in the spirit of perturbation method and

$$
f^{(0)}=\frac{1}{e^{\beta(E(\mathbf{k})-\mu)}+1} .
$$

the Fermi distribution function. Now we consider we have a homogeneous system which means the the distribution function $f$ is indepedent of the position in the system: $\nabla_{x} f=0$.

To the first order approximation

$$
\frac{\partial f}{\partial t}=0
$$

and we have

$$
e \mathbf{E} \cdot\left(\nabla_{p} f^{(0)}\right)=-\frac{f^{(1)}-\bar{f}^{(1)}}{\tau} .
$$

Due to the presence of the applied electric field, the momentum of electrons is no longer isotropic. In the sense of Taylor expansion:

$$
f(x)=f\left(x_{0}\right)+\left.\frac{d f}{d x}\right|_{x=x_{0}}\left(x-x_{0}\right)+\left.\frac{1}{2 !} \frac{d^{2} f}{d x^{2}}\right|_{x=x_{0}}\left(x-x_{0}\right)^{2}+\ldots,
$$

we may think $f^{(1)}$ as an odd function of momentum, therefore $\bar{f}^{(1)}$ is 0 ,

$$
\Rightarrow f^{(1)}=-\tau e \mathbf{E} \cdot\left(\nabla_{p} f^{(0)}\right)
$$

Then we consider the contribution from second-order correction. Since $f^{(0)}$ is time-independent, $f^{(1)}$ is also time-independet as can be seen from the equation above:

$$
\frac{\partial f^{(0)}}{\partial t}=\frac{\partial f^{(1)}}{\partial t}=0
$$

So the $f^{(2)}$ is expected to be the leading-order term in time-dependent contribution. Then the Boltzmann equation now look like

$$
\frac{\partial f^{(2)}}{\partial t}=\tau\left(e \mathbf{E} \cdot \nabla_{p}\right)^{2} f^{(0)}-\frac{f^{(2)}-\bar{f}^{(2)}}{\tau} .
$$


Now taking the angular average of the distribution function

$$
\int \frac{d \hat{p}}{2 \pi}
$$

over every term:

$$
\bar{f}^{(2)}=t \tau \int \frac{\hat{p}}{2 \pi}\left(e \mathbf{E} \cdot \nabla_{p}\right)^{2} f^{(0)}
$$

The second-order contribution in the energy density can be calculated from

$$
\delta \epsilon \equiv N(0) \int_{-\infty}^{\infty} d \epsilon \epsilon \bar{f}^{(2)}
$$

where $N(0)=\frac{2 m V}{\pi}$ is the density of state of a 2-D system at Fermi level (we take $\hbar=1$ ), $V$ is the volume of the system. The reason we use the density of state at fermi level is because we believe that only the electron distribution at the nearby of Fermi surface makes contribution to the change in energy

$$
\begin{aligned}
\delta \epsilon & =N(0) \int_{-\infty}^{\infty} \epsilon t \tau \int \frac{\hat{p}}{2 \pi}\left(e \mathbf{E} \cdot \nabla_{\mathbf{p}}\right)^{2} f^{(0)} \\
& =\frac{V^{2} t}{R}
\end{aligned}
$$

which obviously is the Joule heating.

As we can see that the energy increases linearly with respect to time, so it is impossible

to reach a steady state $\left(\frac{\partial f}{\partial t}=0\right)$ under the assumption there is only elastic scattering process; therefore we have to introduce an inelastic scattering process to remove the heat.

\subsubsection{Summary of Arai's method}

In this section, we will explain the idea of effective temperature and show the electric field temperature dependence at large field which is the feature of nonlinear transport [Arai (1983)]. In the previous section, we see that with elastic scattering process alone system cannot reach a steady state so inelastic scattering process is needed. In the non-equilibrium current carrying state considered, the lowest electron temperature attainable in a biased resistor is given by the lowest temperature where the inelastic scattering is capable of maintaining a steady state. This nonequilibrium noise should be observable for electric fields sm $\backslash$ rangle_\{ $\backslash$ mbox $\{$ inter $\}$ \}all enough that Joule heating is insufficient to drive the phonons out of equilibrium. 
We begin by considering the Boltzmann equation for a simplified model containing the essential physics. The elastic impurity scattering rate $\tau_{i m p}^{-1}$ characterizs the relaxation of the electronic distribution function $f(\mathbf{p})$ to its angular average $\bar{f}(p)$. The inelastic scattering $\tau_{p h}^{-1}$ relaxes the energy by changing the magnitude of the momentum and therefore drives the distribution to thermal equilibrium as represernted by the Fermi distribution $f^{e q}$. The heat bath temperature enters through $f^{e q}$.

Result are obtained by self-consistently solving the Boltzmann equation using an iterative procedure which retains the first two terms of a decomposition of the distribution function into Legendre polynomials (i.e., the $s p$-approximation). This method holds for all two-dimensional system as long as it obeys the transportation model introduced by Arai.

$$
-e \mathbf{E} \cdot \nabla_{p} f(\mathbf{p})=-\frac{1}{\tau_{i m p}}[f(\mathbf{p})-\bar{f}(p)]-\frac{1}{\tau_{p h}}\left[f(\mathbf{p})-f^{e q}(p)\right],
$$

After some algebra, we have the distribution functions $f^{(0)}$ and $f^{(1)}$ :

$$
\begin{aligned}
f^{(0)}(p) & =\int_{-\infty}^{\infty} \frac{v_{F} d p^{\prime}}{2 \pi}\left(\frac{\pi}{e E l^{*}}\right) \exp \left(\frac{-v_{F}\left|p-p^{\prime}\right|}{e E l^{*}}\right) f^{e q}\left(p^{\prime}\right) . \\
f^{(1)}(p) & =\left(\frac{e E l^{*}}{v_{F}}\right)\left(\frac{3 \tau}{\tau_{p h}}\right)^{\frac{1}{2}} \frac{\partial}{\partial p} f^{(0)}(p) .
\end{aligned}
$$

It shows that the distribution function is smeared out on an energy scale given by $e E l^{*}$ with $l^{*}$ the drift distance. This smearing effect dominates the shape of the distribution at low temperatures where $f^{e q}$ is essentially a step function at $p_{F}$.

We can define an effective temperature $T^{*}$ which is defined by assuming the nonequilibrium state as a equilibrium state with temperature $T^{*}$. Effective temperature $T^{*}$ can be done by using the Sommerfeld expansion [Ashcroft and Mermin (1976)]:

$$
k_{B} T^{*}= \begin{cases}k_{B} T & e E l^{*} \ll k_{B} T \\ 0.780 e E l^{*} & e E l^{*} \gg k_{B} T\end{cases}
$$

\subsection{The Schwinger mechanism of pair production}

Schwinger mechanism is a process of particle-antiparticle creation and in the case of graphene, it becomes electron-hole creation. In the conventional way, the canonical momentum $\mathbf{p} \rightarrow \mathbf{p}-\frac{e \mathbf{A}}{c}$ 
is used to solve this term. However, as $t \rightarrow \infty$, it can be simplified to Landau-Zener dynamics which describes the transition rate between two states in a nonadiabatic process in the long time limit. The schematic description is shown in Fig. 3.1 [Dora and Moessner (2010)].

The special feature of Dirac electrons relevant for transport in finite field include: (i) their velocity is pinned to the "light cone" fermi velocity, $v_{F}$, (ii) relativistic particles undergo pair production in strong electric fields, as predicted by Schwinger, and (iii) a uniform electric field modifies locally the geometry of the Fermi surface by moving the Dirac point around in momentum space 3.26 .

The Dirac equation of graphene near the Dirac point (the Fermi energy point) with time dependent vector potential $\mathbf{A}=-\mathbf{E} t$ in $k_{x^{-}}$direction can be described as:

$$
\begin{gathered}
H=v\left[\sigma_{x}\left(p_{x}-e A(t)\right)+\sigma_{y} p_{y}\right] \\
i \hbar \partial_{t} \Psi_{p}(t)=H \Psi_{p}(t)
\end{gathered}
$$

with $v \sim 10^{6} \mathrm{~m} / \mathrm{s}$ the velocity of electron in graphene. Now we perform a time dependent unitary transformation to diagonalize the Hamiltonian:

$$
\begin{cases}H & \Rightarrow U^{\dagger} H U \\ \Psi & \Rightarrow U^{\dagger} \Psi \equiv \Phi\end{cases}
$$

and the $U$ can be expressed as

$$
\frac{1}{\sqrt{2}}\left(\begin{array}{cc}
e^{-\frac{i \varphi}{2}} & e^{-\frac{i \varphi}{2}} \\
e^{\frac{i \varphi}{2}} & -e^{\frac{i \varphi}{2}}
\end{array}\right)
$$

since a $2 \times 2$ unitary matrix is isomorphic to $\mathrm{U}(1)$ group, there can be only one variable which we call it $\varphi$.

$$
U^{\dagger} H U=v\left(\begin{array}{cc}
\left(p_{x}-e A(t)\right) \cos \varphi+p_{y} \sin \varphi & -i\left(p_{x}-e A(t)\right) \sin \varphi+i p_{y} \cos \varphi \\
i\left(p_{x}-e A(t)\right) \sin \varphi-i p_{y} \cos \varphi & -\left(p_{x}-e A(t)\right) \cos \varphi-p_{y} \sin \varphi
\end{array}\right)
$$

With the new Hamiltonian being diagonalized, $\varphi$ must be

$$
\tan \varphi=\frac{p_{y}}{p_{x}-e A(t)}
$$


this also means that we have

$$
\begin{aligned}
\sin \varphi & =\frac{p_{y}}{\sqrt{\left(p_{x}-e A(t)^{2}+p_{y}^{2}\right.}} \\
\cos \varphi & =\frac{p_{x}-e A(t)}{\sqrt{\left(p_{x}-e A(t)^{2}+p_{y}^{2}\right.}}
\end{aligned}
$$

and

$$
U^{\dagger} H U=v\left(\begin{array}{cc}
\sqrt{\left(p_{x}-e A(t)^{2}+p_{y}^{2}\right.} & 0 \\
0 & -\sqrt{\left(p_{x}-e A(t)^{2}+p_{y}^{2}\right.}
\end{array}\right)
$$

While the left hand side of 3.20 can be written as

$$
i \hbar \partial_{t}\left(U \Phi_{p}(t)\right)=i \hbar\left(\frac{\partial U}{\partial t}\right) \Phi_{p}(t)+U H \Psi_{p}(t)
$$

since we already know the second term we only need to focus on the first term:

$$
i \hbar\left(\frac{\partial U^{\dagger}}{\partial t}\right)=-\frac{\hbar}{2} \frac{\partial \varphi}{\partial t} U^{\dagger}
$$

From trigonometry, we have

$$
\frac{\partial \varphi}{\partial t}=\frac{p_{y} e E}{\left(p_{x}-e A(t)\right)^{2}+p_{y}^{2}}
$$

therefore we may obtain

$$
\begin{aligned}
i \hbar \partial_{t} \Phi_{p}(t) & =\left[\sigma_{z} \epsilon_{p}(t)-\sigma_{x} \frac{\hbar v^{2} p_{y} e E}{2 \epsilon_{p}^{2}(t)}\right] \Phi_{p}(t) \\
\Phi_{p}(t=0) & =\left(\begin{array}{l}
0 \\
1
\end{array}\right)
\end{aligned}
$$

assuming that at $t=0$ the system is the lower state and $\epsilon_{p}(t)=v \sqrt{\left(p_{x}-e A(t)\right)^{2}+p_{y}^{2}}$. In Dirac equation the current density operator is

$$
j=-e v \sigma
$$

as shown in section 2.2. So the current density is 


$$
\left\langle j_{x}\right\rangle_{p}(t)=-e v\left[\cos \varphi\left(|\alpha(t)|^{2}-|\beta(t)|^{2}\right)+2 \sin \varphi \Re\left(i \alpha_{p}(t) \beta_{p}^{*}(t)\right)\right]
$$

The first term is the current from carries: electron for upper band and holes for lower band as indicated by the minus sign. The second one describes electron transition between the upper band and the lower band, and is responsible for Zitterbewegung.

By using 3.30, we have

$$
i \hbar \frac{\partial \alpha}{\partial t}=\epsilon_{p} \alpha-\frac{\hbar v^{2} p_{y} e E}{2 \epsilon_{p}^{2}(t)} \beta
$$

We can multiply both side of 3.33 by $\alpha^{*}$ and take the real part,

$$
\Re\left(\alpha^{*} \hbar \frac{\partial \alpha}{\partial t}\right)=\Re\left(-i \epsilon_{p} \alpha^{*} \alpha+i \frac{\hbar v_{F}^{2} p_{y} e E}{2 \epsilon_{p}^{2}(t)} \alpha^{*} \beta\right)
$$

since $\frac{\partial|\alpha|^{2}}{\partial t}=2 \Re\left(\alpha_{p}(t) \partial_{t} \alpha_{p}^{*}(t)\right)=2 \Re\left(\alpha_{p}^{*}(t) \partial_{t} \alpha_{p}(t)\right)$,

$$
\begin{aligned}
\frac{\hbar}{2} \frac{\partial|\alpha|^{2}}{\partial t} & =\frac{\hbar v^{2} p_{y} e E}{2 \epsilon_{p}^{2}(t)} \Re\left(i \alpha_{p}^{*} \beta_{p}\right) \\
& =-\frac{\hbar v^{2} p_{y} e E}{2 \epsilon_{p}^{2}(t)} \Re\left(i \alpha_{p} \beta_{p}^{*}\right)
\end{aligned}
$$

thus the current density 3.32 becomes

$$
\left\langle j_{x}\right\rangle_{p}(t)=-e v_{F}\left[\frac{v\left(p_{x}-e E t\right)}{\epsilon_{p}(t)}\left(2 n_{p}(t)-1\right)-2 \frac{\epsilon_{p}(t)}{v e E} \partial_{t} n_{p}(t)\right]
$$

with $n_{p}(t)=|\alpha(t)|^{2}$. In condensed matter, the first term and second term in 3.36 are called as intraband and interband current, respectively. As we can see, the first term vanishes at half filling after integrating over momentum because the equal amount of electrons and holes move in the same direction: one electron with momentum $\mathbf{k}$ excited to upperband creating one hole with momentum $\mathbf{k}$ in the lower band. The mechanism is similar to Schwinger's pair production, which describes the simultaneous creation of particle-antiparticle pairs; whereas in graphene, it is electron-hole pairs.

Here we discuss the current density behavior with different magnitude of applied field $\mathbf{E}$. First case we set the field very small compared to the electron momentum $p$ and we obtain

$$
i \hbar \frac{\partial}{\partial t}\left(\begin{array}{c}
\alpha \\
\beta
\end{array}\right)=\left(\begin{array}{cc}
v p & -\frac{\hbar p_{y} e E}{2 p^{2}} \\
-\frac{\hbar p_{y} e E}{2 p^{2}} & -v p
\end{array}\right)\left(\begin{array}{l}
\alpha \\
\beta
\end{array}\right)
$$


which is nothing else but an eigenvalue problem with initial condition $\alpha(t=0)=0$ and $\beta(t=0)=1$. We can get

$$
\begin{aligned}
& \alpha(t) \sim \alpha_{0} \sin v p t \\
& \beta(t) \sim \beta_{0} \cos v p t
\end{aligned}
$$

with $\beta_{0} \sim 1$ and $\alpha_{0} \sim \frac{\hbar p_{y} e E}{2 p^{3} v}$ as the eigenstate. This result is as expected because the small electric field cannot produce significant amount of electron-hole pairs. Therefore

$$
\begin{aligned}
n_{p}(t) & =|\alpha(t)|^{2} \\
& =\frac{\left(e E \hbar p_{y}\right)^{2}}{4 v^{2}|p|^{6}} \sin ^{2}\left(\frac{v|p| t}{\hbar}\right)
\end{aligned}
$$

As the electric field is sufficiently small, we can ignore the first term in 3.36 because it is proportional to $E^{2}$ and the effect is suppressed by the linear term $\frac{e v^{2}\left(p_{x}-e E t\right)}{\epsilon_{p}(t)}(-1)$; thus we only need to consider the intraband current which describes intraband transition. As we want to know the total current density, we need to sum up all the contribution from carriers:

$$
\begin{aligned}
\left\langle j_{x}\right\rangle & =\frac{4}{V} v e \sum_{\mathbf{k}} \frac{\mathbf{k}}{|\mathbf{k}|} \frac{2 v p}{v e E} \partial_{t} n_{p}(t) \\
& =\frac{\pi}{2} \frac{e^{2} E}{h}
\end{aligned}
$$

where the 4 comes electron degeneracy in graphene. The constant conductivity

$$
\sigma=\frac{\pi}{2} \frac{e^{2}}{h}
$$

is the minimal conductivity [Lewkowicz and Rosenstein (2009)].

At long time limit, the pair-production $n_{p}(t)$ obtained from Landau-Zener dynamics [Green and Sondhi $(2005)]$ is

$$
n_{p}(t)=\Theta\left(p_{x}\right) \Theta\left(e E t-p_{x}\right) \exp \left[-\frac{\pi v p_{y}^{2}}{e E \hbar}\right]
$$

which is the celebrated pair production rate by Schwinger [Schwinger (1951); Tanji (2009)]. The current due to this pair-production $n_{p}(t)$ is

$$
\begin{aligned}
\left\langle j_{x}\right\rangle_{\text {Schwinger }} & =e v \sum_{\lambda= \pm} \int d p_{x} d p_{y} n_{p}(t) \\
& =\frac{2 e^{2} E^{\frac{3}{2}} t}{\hbar^{2}} \sqrt{e v \hbar}
\end{aligned}
$$


which is the result of Schwinger/Kibble-Zurek mechanism. While for the interband current

$$
\left\langle j_{x}\right\rangle_{\text {inter }}=\frac{2 e^{2}}{\pi^{3} \hbar} E
$$

which corresponds to Kubo mechanism and is overwhelmed by the intraband current at large

field limit. Here we can see that $\sigma_{x x} \sim E^{\frac{1}{2}}$ which demostrates nonlinear transport in graphene at large electric field.

While the pair-production rate $S_{\mathbf{k}}$ can be written as

$$
S_{\mathbf{k}}=\frac{d n_{p}(t)}{d t}=\delta\left(\frac{\hbar k_{x}}{e E}\right) \exp \left[-\frac{\pi v_{e} \hbar k_{y}^{2}}{e E}\right]
$$

As refering back to 3.2 , we see that we need to put $S_{\mathbf{k}}$ in the right hand side of the equation because in this case, the change in distribution function is not only determined by the collision integral, but also affected by the pair-production.

\subsection{Electron-phonon coupling in graphene}

\subsubsection{The free phonon part}

The free phonon part of the Hamiltonian is given as

$$
H_{0, \mathrm{ph}}=\sum_{\mathbf{q} a} \hbar \omega_{\mathbf{q} a} b_{\mathbf{q}, a}^{\dagger} b_{\mathbf{q}, a}
$$

where $\omega_{\mathbf{q}}$ is the phonon frequency for the phonon branch $a$.

\subsubsection{Electron-phonon interaction}

One of the common scattering potentials is the oscillating potential produced by lattice vibrations. This potential can be written as

$$
\begin{aligned}
U_{S} & =D_{A} \frac{\partial u_{\mathbf{q}}}{\partial x} \\
& =\frac{D_{A} q}{\sqrt{2 \rho \omega_{\mathbf{q}}}}\left(\hat{b}_{\mathbf{q}}^{\dagger} e^{-i(\mathbf{q} \cdot \mathbf{r}-\omega t)}+\hat{b}_{\mathbf{q}} e^{i(\mathbf{q} \cdot \mathbf{r}-\omega t)}\right)
\end{aligned}
$$

where $K_{\mathbf{q}}=D_{A}|\mathbf{q}|$ is related to the deformation potential $D_{A}$ due to a perturbed lattice constants and band structure, and $u_{\mathbf{q}}=A_{\mathbf{q}} e^{i(\mathbf{q} \cdot \mathbf{r}-\omega t)}+A_{\mathbf{q}}^{\dagger} e^{-i(\mathbf{q} \cdot \mathbf{r}-\omega t)}$ with $A_{\mathbf{q}}=\sqrt{\frac{1}{2 \rho \omega_{\mathbf{q}}}} \hat{b}_{\mathbf{q}}$ and 
$A_{\mathbf{q}}^{\dagger}=\sqrt{\frac{1}{2 \rho \omega_{\mathbf{q}}}} \hat{b}_{\mathbf{q}}^{\dagger}$. The overall potential contributed by all the vibrational modes is therefore

$$
\begin{aligned}
U & =\sum_{\mathbf{q}} U_{s} \\
& =\sum_{\mathbf{q}} \frac{D_{A} q}{\sqrt{2 \rho \omega_{\mathbf{q}}}}\left(\hat{b}_{\mathbf{q}}^{\dagger} e^{-i(\mathbf{q} \cdot \mathbf{r}-\omega t)}+\hat{b}_{\mathbf{q}} e^{i(\mathbf{q} \cdot \mathbf{r}-\omega t)}\right)
\end{aligned}
$$

In the electron-phonon interaction, potential $U$ is regarded as an one-body operator: one electron comes in, interacts with phonon, then goes out. Therefore the Hamiltonian $H_{1}$ of electronphonon interaction can be written as

$$
\begin{aligned}
H_{1} & =\sum_{k, k^{\prime}}\left\langle k|U| k^{\prime}\right\rangle a_{k}^{\dagger} a_{k^{\prime}} \\
& =\sum_{\mathbf{k}, \mathbf{q}} \frac{D_{A} q}{\sqrt{2 \rho \omega_{\mathbf{q}}}} a_{\mathbf{k}+\mathbf{q}}^{\dagger} a_{\mathbf{k}}\left(\hat{b}_{-\mathbf{q}}^{\dagger}+\hat{b}_{\mathbf{q}}\right)
\end{aligned}
$$

with $a^{\dagger}$ and $a$ electron creation operator and annihilation operator respectively and $k=\left(k^{0}, \mathbf{k}\right)$. This result holds for both sublattice blue and sublattice red as well as is diagonalized. Here we can use the symbols introduced in 2.19 and 2.20 without changing the Hamiltonian $H_{1}$, so finally we get [Prange-1964]

$$
H_{1}=\sum_{\mathbf{k}, \mathbf{q}} \sum_{i=1}^{2} \psi_{i}^{\dagger}(\mathbf{k}+\mathbf{q})\left(b_{-\mathbf{q}}^{\dagger}+b_{\mathbf{q}}\right) I \psi_{i}(\mathbf{k})
$$

where $I$ is a $2 \times 2$ identity matrix.

After the proper unitary transformation $U$ which diagonalizes electron bare Hamiltonian, the electron phonon interaction can in gerenal be written as Prange and Kadanoff (1964)

$$
H_{e-p h}=\sum_{\mathbf{k}, \mathbf{k}^{\prime}, \lambda \lambda^{\prime} a} g_{\mathbf{k}, \mathbf{k}^{\prime}}^{\lambda \lambda^{\prime}, a} \gamma_{\mathbf{k}, \lambda}^{\dagger} \gamma_{\mathbf{k}^{\prime}, \lambda^{\prime}}\left(b_{\mathbf{k}^{\prime}-\mathbf{k}, a}^{\dagger}+b_{\mathbf{k}-\mathbf{k}^{\prime}, a}\right)
$$

where $g_{\mathbf{k}, \mathbf{k}^{\prime}}^{\lambda \lambda^{\prime}, a}$ is the electron-phonon matrix element between electrons in states $(\mathbf{k}, \lambda)$ and $\left(\mathbf{k}^{\prime}, \lambda^{\prime}\right)$ with phonon mode $a$ where the phonon momentum $\mathbf{q}=\mathbf{k}^{\prime}-\mathbf{k}$ is determined by momentum conservation:

$$
g_{\mathbf{k}, \mathbf{k}^{\prime}}^{\lambda \lambda^{\prime}, a}=\frac{D q}{\sqrt{2 \rho_{m} \omega_{\mathbf{q}}}}\left\langle\mathbf{k} \lambda \mid \mathbf{k}^{\prime} \lambda^{\prime}\right\rangle
$$




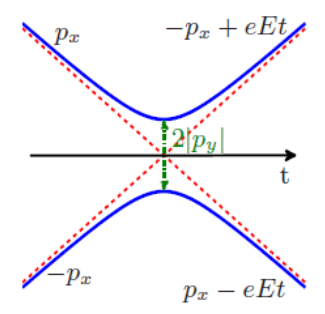

Figure 3.1 Schematic description of temporal evolution of Landau-Zener dynamics. For any arbitrary point in $p$-space, the energy difference between the corresponding two points in the upper and lower band is $\Delta E=2 p v_{F}$. As the time evolves, their energies are getting closer and closer, and reaching the closest distance $\Delta E_{\min }=2 p_{y} v_{F}$ at $t^{*}=\frac{p_{x}}{e E}$; then they move away from each other. The nonadiabatic process induces a transition between the two bands until the transition is completed when the two states are far from each other. In our case we choose at, all the electrons lie in the lower band.

Table 3.1 Symbols and their corresponding physical meanings in a phonon scattering potential $U_{S}=D_{A} \frac{\partial u}{\partial x}=K_{\mathbf{q}} A_{\mathbf{q}} e^{ \pm i(\mathbf{q} \cdot \mathbf{x}-\omega t)}$

\begin{tabular}{|c|c|}
\hline Symbol & Physical meaning \\
\hline \hline$D_{A}$ & lattice deformation potential \\
\hline$K_{\mathbf{q}}$ & $D_{A}|\mathbf{q}|$ \\
\hline$\hat{b}_{\mathbf{q}}^{\dagger}$ & phonon creation operator \\
\hline$\hat{b}_{\mathbf{q}}$ & phonon annihilation operator \\
\hline $\mathbf{q}$ & momentum of phonon \\
\hline$\omega$ & energy of single phonon with speed of light $c=1$ \\
\hline
\end{tabular}


where $\rho_{m} \simeq 0.76 \mathrm{mg} / \mathrm{m}^{2}$ is the mass density of graphene and values for the deformation coupling constant are in the range $D \simeq 10-30 \mathrm{eV}$ [Hwang-2008]. For the matrix element follows

$$
\begin{aligned}
\left\langle\mathbf{k} \lambda \mid \mathbf{k}^{\prime} \lambda^{\prime}\right\rangle & =\left(U_{\mathbf{k}} U_{\mathbf{k}^{\prime}}^{-1}\right)_{\lambda \lambda^{\prime}} \\
& =\frac{1+\lambda \lambda^{\prime}}{2} \cos \left(\frac{\theta_{\mathbf{k}}-\theta_{\mathbf{k}^{\prime}}}{2}\right)-i \frac{1-\lambda \lambda^{\prime}}{2} \sin \left(\frac{\theta_{\mathbf{k}}-\theta_{\mathbf{k}^{\prime}}}{2}\right)
\end{aligned}
$$

which yields

$$
\begin{aligned}
\left|\left\langle\mathbf{k} \lambda \mid \mathbf{k}^{\prime} \lambda^{\prime}\right\rangle\right|^{2} & =\frac{1+\lambda \lambda^{\prime} \cos \left(\theta_{\mathbf{k}}-\theta_{\mathbf{k}^{\prime}}\right)}{2} . \\
e^{i \theta_{\mathbf{k}}} & =\frac{k_{x}+i k_{y}}{k} \\
e^{i \theta_{\mathbf{k}+\mathbf{q}}} & =\frac{k_{x}+q_{x}+i\left(k_{y}+q_{y}\right)}{\sqrt{k+q+2 \mathbf{k} \cdot \mathbf{q}}} \\
\cos \left(\theta_{\mathbf{k}}-\theta_{\mathbf{k}^{\prime}}\right) & =\Re\left[e^{i \theta_{\mathbf{k}}} e^{\left.-i \theta_{\mathbf{k}+\mathbf{q}}\right]}\right. \\
& =\frac{\mathbf{k} \cdot(\mathbf{k}+\mathbf{q})}{k|\mathbf{k}+\mathbf{q}|}
\end{aligned}
$$

\subsubsection{Collision operator due to electron-phonon coupling}

We consider the quantum Boltzmann equation of fermions with distribution function $f_{\lambda}(\mathbf{k}, t)$

$$
\frac{\partial f_{\mathbf{k} \lambda}(t)}{\partial t}+\frac{e \mathbf{E}}{\hbar} \cdot \frac{\partial f_{\mathbf{k} \lambda}(t)}{\partial \mathbf{k}}=I_{\mathbf{k} \lambda}(t)
$$

and the collision integral due to electron-phonon coupling can be written as

$$
\begin{aligned}
I_{\mathbf{k} \lambda}= & -2 \pi \int_{\mathbf{k}^{\prime}, \lambda^{\prime}}\left|g_{\mathbf{k}, \mathbf{k}^{\prime}}^{\lambda \lambda^{\prime}, a}\right|^{2}\left[f_{\mathbf{k} \lambda}\left(1-f_{\mathbf{k}^{\prime}, \lambda^{\prime}}\right)+\left(f_{\mathbf{k} \lambda}-f_{\mathbf{k}^{\prime}, \lambda^{\prime}}\right) n_{\mathbf{q}, a}\right] \\
& \times \delta\left(\varepsilon_{\mathbf{k} \lambda}-\omega_{\mathbf{k}-\mathbf{k}^{\prime}, a}-\varepsilon_{\mathbf{k}^{\prime}, \lambda^{\prime}}\right) \\
& +2 \pi \int_{\mathbf{k}^{\prime}, \lambda^{\prime}}\left|g_{\mathbf{k}, \mathbf{k}^{\prime}}^{\lambda \lambda^{\prime}, a}\right|^{2}\left[f_{\mathbf{k}^{\prime}, \lambda^{\prime}}\left(1-f_{\mathbf{k} \lambda}\right)-\left(f_{\mathbf{k} \lambda}-f_{\mathbf{k}^{\prime}, \lambda^{\prime}}\right) n_{\mathbf{q}, a}\right] \\
& \times \delta\left(\varepsilon_{\mathbf{k} \lambda}+\omega_{\mathbf{k}-\mathbf{k}^{\prime}, a}-\varepsilon_{\mathbf{k}^{\prime}, \lambda^{\prime}}\right)
\end{aligned}
$$

\subsection{Solution of the linearized Boltzmann equation}

Next we insert the small electric field expansion into the collision integral, i.e. we consider

$$
f_{\mathbf{k} \lambda}=f_{\mathbf{k} \lambda}^{0}+e \lambda \frac{\mathbf{E} \cdot \mathbf{k}}{k} f_{\mathbf{k} \lambda}^{0}\left(1-f_{\mathbf{k} \lambda}^{0}\right) g_{\lambda}(k) .
$$


where $f_{\mathbf{k} \lambda}^{0}=\left(e^{\beta v \lambda k}+1\right)^{-1}$. Note that

$$
f_{\mathbf{k} \lambda}^{0}\left(1-f_{\mathbf{k} \lambda}^{0}\right)=\frac{e^{\beta v k}}{\left(e^{\beta v k}+1\right)^{2}}
$$

is in fact independent on $\lambda$. It then follows that

$$
\begin{aligned}
I_{\mathbf{k} \lambda}= & -2 \pi e \int_{\mathbf{k}^{\prime}, \lambda^{\prime}}\left|g_{\mathbf{k}, \mathbf{k}^{\prime}}^{\lambda \lambda^{\prime}, a}\right|^{2}\left[f_{\mathbf{k} \lambda}^{(0)}\left(-\lambda^{\prime} \frac{\mathbf{E} \cdot \mathbf{k}^{\prime}}{k^{\prime}}\right) f_{\mathbf{k}^{\prime} \lambda^{\prime}}^{0}\left(1-f_{\mathbf{k}^{\prime} \lambda^{\prime}}^{0}\right) g_{\lambda^{\prime}}\left(k^{\prime}\right)\right. \\
& \left.+\left(1-f_{\mathbf{k}^{\prime}, \lambda^{\prime}}^{(0)}\right) \lambda \frac{\mathbf{E} \cdot \mathbf{k}}{k} f_{\mathbf{k} \lambda}^{0}\left(1-f_{\mathbf{k} \lambda}^{0}\right) g_{\lambda}(k)\right] \\
& \times\left[\left(1+n_{\mathbf{q}, a}\right) \delta\left(\varepsilon_{\mathbf{k} \lambda}-\omega_{\mathbf{q}, a}-\varepsilon_{\mathbf{k}^{\prime}, \lambda^{\prime}}\right)+n_{\mathbf{q}, a} \delta\left(\varepsilon_{\mathbf{k} \lambda}+\omega_{\mathbf{q}, a}-\varepsilon_{\mathbf{k}^{\prime}, \lambda^{\prime}}\right)\right] \\
& +2 \pi e \int_{\mathbf{k}^{\prime}, \lambda^{\prime}}\left|g_{\mathbf{k}, \mathbf{k}^{\prime}}^{\lambda \lambda^{\prime}}\right|^{2}\left[f_{\mathbf{k}^{\prime} \lambda^{\prime}}^{(0)}\left(-\lambda \frac{\mathbf{E} \cdot \mathbf{k}}{k}\right) f_{\mathbf{k} \lambda}^{0}\left(1-f_{\mathbf{k} \lambda}^{0}\right) g_{\lambda}(k)\right. \\
& \left.+\left(1-f_{\mathbf{k} \lambda}^{(0)}\right) \lambda \frac{\mathbf{E} \cdot \mathbf{k}^{\prime}}{k^{\prime}} f_{\mathbf{k}^{\prime} \lambda^{\prime}}^{0}\left(1-f_{\mathbf{k}^{\prime} \lambda^{\prime}}^{0}\right) g_{\lambda^{\prime}}\left(k^{\prime}\right)\right] \\
& \times\left[n_{\mathbf{q}, a} \delta\left(\varepsilon_{\mathbf{k} \lambda}-\omega_{\mathbf{q}, a}-\varepsilon_{\mathbf{k}^{\prime}, \lambda^{\prime}}\right)+\left(1+n_{\mathbf{q}, a}\right) \delta\left(\varepsilon_{\mathbf{k} \lambda}+\omega_{\mathbf{q}, a}-\varepsilon_{\mathbf{k}^{\prime}, \lambda^{\prime}}\right)\right]
\end{aligned}
$$

\subsubsection{Coupling to longitudinal accustic phonons}

In case of longitudinal acustic phonons, we have

$$
\begin{aligned}
\left|g_{\mathbf{k}, \mathbf{k}^{\prime}}^{\lambda \lambda^{\prime}, a}\right|^{2} & =\frac{D^{2}\left(\mathbf{k}-\mathbf{k}^{\prime}\right)^{2}}{2 \rho_{m} \omega_{\mathbf{k}-\mathbf{k}^{\prime}}}\left|\left\langle\mathbf{k} \lambda \mid \mathbf{k}^{\prime} \lambda^{\prime}\right\rangle\right|^{2} \\
& =\frac{D^{2}\left(\mathbf{k}-\mathbf{k}^{\prime}\right)^{2}}{2 \rho_{m} \omega_{\mathbf{k}-\mathbf{k}^{\prime}}} \frac{1+\lambda \lambda^{\prime} \cos \left(\theta_{\mathbf{k}}-\theta_{\mathbf{k}^{\prime}}\right)}{2} .
\end{aligned}
$$

The phonon frequency is $\omega_{q}=c q$ and we take the limit $c / v \ll 1$. To leading order, this implies that we can neglect the phonon frequencies $\omega_{\mathbf{q}}$ in the delta functions that guarantee energy coservation. This in turn implies that we can restrict ourselves to intraband scattering processes with $\lambda^{\prime}=\lambda$. Finally, for the bose-factor follows in the limit $c / v \ll 1$ that

$$
n_{\mathbf{q}} \sim 1+n_{\mathbf{q}} \sim \frac{T}{c q}
$$

and under this limit, the angular dependence only shows on $\left|g_{\mathbf{k}, \mathbf{k}^{\prime}}^{\lambda \lambda^{\prime}, a}\right|^{2}$ and $\mathbf{E} \cdot \mathbf{k}^{\prime}$. This leads us to an identity equation

$$
\int d \theta_{\mathbf{k}^{\prime}}\left|g_{\mathbf{k}, \mathbf{k}^{\prime}}^{\lambda \lambda^{\prime}, a}\right|^{2} \frac{\mathbf{E} \cdot \mathbf{k}^{\prime}}{k^{\prime}}=\int d \theta_{\mathbf{k}^{\prime}}\left|g_{\mathbf{k}, \mathbf{k}^{\prime}}^{\lambda \lambda^{\prime}, a}\right|^{2} \frac{(\mathbf{E} \cdot \mathbf{k})\left(\mathbf{k} \cdot \mathbf{k}^{\prime}\right)}{k^{2} k^{\prime}}
$$


It follows that

$$
\begin{aligned}
I_{\mathbf{k} \lambda}= & \frac{4 \pi e T \mathbf{E} \cdot \mathbf{k} \lambda}{k c} \int_{\mathbf{k}^{\prime}} \mid g_{\mathbf{k}, \mathbf{k}^{\prime}}^{\lambda \lambda,\left.\right|^{2}} \frac{g_{\lambda}(k)}{q}\left[\left(\frac{\mathbf{k} \cdot \mathbf{k}^{\prime}}{k k^{\prime}}\right) f_{\mathbf{k}^{\prime} \lambda}^{0}\left(1-f_{\mathbf{k}^{\prime} \lambda}^{0}\right)-f_{\mathbf{k} \lambda}^{0}\left(1-f_{\mathbf{k} \lambda}^{0}\right)\right] \\
& \times\left[\delta\left(\varepsilon_{\mathbf{k}}-\varepsilon_{\mathbf{k}^{\prime}}\right)\right]
\end{aligned}
$$

Since the energy of electron is much larger than that of phonon, we may use the quasielastic scattering process, i.e. $\delta\left(\varepsilon_{\mathbf{k} \lambda}-\varepsilon_{\mathbf{k}^{\prime}, \lambda}\right)=\delta\left(v k-v k^{\prime}\right)$, to obtain the collision integral. Since $f_{\mathbf{k} \lambda}^{0}$ only depends on $|\mathbf{k}|$, it further follows that $f_{\mathbf{k} \lambda}^{0}=f_{\mathbf{k}^{\prime} \lambda}^{0}=f^{0}(v \lambda k)$. Inserting the electron phonon scattering element yields

$$
I_{\mathbf{k} \lambda}=-\frac{e T D^{2} \mathbf{E} \cdot \mathbf{k} \lambda}{4 \rho_{m} c^{2} v} g_{\lambda}(k) f_{\mathbf{k} \lambda}^{0}\left(1-f_{\mathbf{k} \lambda}^{0}\right)
$$

Inserting this result into the Boltzmann equation, 3.60, yields

$$
-e \mathbf{E} \cdot \frac{\mathbf{k}}{k} \frac{e^{\beta v k}}{\left(e^{\beta v k}+1\right)^{2}} \beta v \lambda=-2 \pi e \frac{\mathbf{E} \cdot \mathbf{k}}{k} \lambda \frac{D^{2} T}{8 \pi \rho_{m} v c^{2}} k \frac{e^{\beta v k}}{\left(e^{\beta v k}+1\right)^{2}} g_{\lambda}(k)
$$

which finally yields

$$
g_{\lambda}(k)=\frac{4 \rho_{m} c^{2}}{D^{2}} \frac{v^{2}}{T^{2} k}
$$

If we insert this resul into 2.44 for the conductivity we find

$$
\begin{aligned}
\sigma & =e^{2} v 2 \pi \frac{4 \rho_{m} c^{2}}{D^{2}} \frac{v}{T} N \int_{0}^{\infty} d x \frac{e^{x}}{\left(e^{x}+1\right)^{2}} \\
& =e^{2} \frac{4 \pi \rho_{m} c^{2} N}{D^{2}} \frac{v^{2}}{T}
\end{aligned}
$$

reintroducing proper units yields

$$
\sigma=N \frac{e^{2}}{\hbar} \frac{\theta_{0}}{T}
$$

with the temperature scale

$$
\theta_{0}=\frac{4 \pi \hbar^{2} \rho_{m} c^{2} v^{2}}{k_{B} D^{2}}
$$

whose numerical value is $3 \times 10^{5} \mathrm{~K}$ for $D=20 \mathrm{eV}$. The result for conductivity measurement [Hwang and Sarma (2008)] turns out to be the same as our result. 


\subsubsection{Relaxation rate}

The natural definition to introduce a relaxation time $\tau$ is via

$$
\frac{d f_{\mathbf{k} \lambda}}{d t}=-\frac{1}{\tau}\left(f_{\mathbf{k} \lambda}-f_{\mathbf{k} \lambda}^{0}\right)
$$

which yields

$$
\tau^{-1}=-\frac{I_{\mathbf{k} \lambda}}{f_{\mathbf{k} \lambda}-f_{\mathbf{k} \lambda}^{0}} .
$$

In our case this corresponds to (introducing proper units)

$$
\tau^{-1}=\frac{D^{2} k_{B} T}{\hbar^{2} 4 \rho_{m} v c^{2}} k=\pi \frac{T}{\theta_{0}} v k
$$

which agrees with the result of [Hwang and Sarma (2008); Stauber et al. (2007)]. 


\section{CHAPTER 4. Scaling theory}

such that the new action $S$ looks like

$$
\begin{aligned}
S= & \frac{1}{b^{2}} \int_{0}^{\Lambda} \frac{k^{\prime} d k^{\prime}}{(2 \pi)^{2}} T \sum_{m, \lambda}\left(-i \omega_{m}+\lambda v \frac{k^{\prime}}{b} \hbar\right) \gamma_{\lambda}^{\dagger}\left(\frac{\mathbf{k}^{\prime}}{b}, \omega_{m}\right) \gamma_{\lambda}\left(\frac{\mathbf{k}^{\prime}}{b}, \omega_{m}\right) \\
& +\frac{1}{b^{2}} \int_{0}^{\Lambda} \frac{q^{\prime} d q^{\prime}}{(2 \pi)^{2}} T \sum_{m}\left(-i \omega_{m}+c \frac{q}{b} \hbar\right) d^{\dagger}\left(\frac{\mathbf{q}^{\prime}}{b}, \omega_{m}\right) d\left(\frac{\mathbf{q}^{\prime}}{b}, \omega_{m}\right) \\
& +\frac{1}{b^{4}} \int_{0}^{\Lambda} \frac{k^{\prime} d k^{\prime}}{(2 \pi)^{2}} \int_{0}^{\Lambda} \frac{q^{\prime} d q^{\prime}}{(2 \pi)^{2}} T^{2} \sum_{m, n, \lambda} g \sqrt{\frac{q^{\prime}}{b}}\left\langle\mathbf{k}^{\prime}, \lambda \mid \mathbf{k}^{\prime}+\mathbf{q}^{\prime}, \lambda\right\rangle \\
& \times \gamma_{\lambda}^{\dagger}\left(\frac{\mathbf{k}^{\prime}}{b}, \omega_{m}\right) \gamma_{\lambda}\left(\frac{\mathbf{k}^{\prime}+\mathbf{q}^{\prime}}{b}, \omega_{n}\right)\left[d^{\dagger}\left(\frac{\mathbf{q}^{\prime}}{b}, \omega_{n-m}\right)+d\left(-\frac{\mathbf{q}^{\prime}}{b}, \omega_{m-n}\right)\right]
\end{aligned}
$$

Here we assume that both $\gamma\left(\frac{\mathbf{k}^{\prime}}{b}, \omega_{m}\right)$ and $d\left(\frac{\mathbf{q}^{\prime}}{b}, \omega_{m}\right)$ scale as

$$
\begin{aligned}
& \gamma\left(\frac{\mathbf{k}^{\prime}}{b}, \omega_{m}\right)=b^{\rho}\left(\mathbf{k}, \omega_{m}^{\prime}\right) \\
& d\left(\frac{\mathbf{q}^{\prime}}{b}, \omega_{m}\right)=b^{\rho}\left(\mathbf{k}, \omega_{m}^{\prime}\right)
\end{aligned}
$$

since their bare Hamiltonians look the same, they must obey the same scaling law. Then we assume that

$$
\omega_{m}^{\prime}=b^{\zeta} \omega_{m}
$$

which implies

$$
T^{\prime}=b^{\zeta} T
$$

from their relation shown in Matsubara frequency. In renormalization group approach, it is expected that the action is independent of scaling. So $\omega_{m}$ and $k$ must share the same scaling law:

$$
\zeta=1
$$


then we may obtain

$$
\rho=2
$$

from either the bare electron action and bare phonon action.

Let's move to electron-phonon interaction term to find out the exponent $\eta$ of coupling constant $g$ :

$$
g^{\prime}=b^{\eta} g
$$

it is straightforward to find that

$$
\eta=-\frac{1}{2}
$$

In the end, although we may use the canonical momentum to find that $\mathbf{E}^{\prime}=b^{2} \mathbf{E}$. I think it is easier to use Coulomb electric field to obtain the same conclusion:

$$
\mathbf{E}=\frac{e^{2}}{r^{2}} \hat{r}
$$

then since $r$ scales as $1 / k$, so

$$
\frac{1}{r^{2}}=\frac{b^{2}}{r^{\prime 2}}
$$

then

$$
\mathbf{E}=b^{2} \mathbf{E}^{\prime}
$$

Thus in the finite electric field, we have

$$
S_{\frac{\Lambda}{b}}(E, T, g)=S_{\Lambda}\left(b^{2} E, b T, b^{-\frac{1}{2}} g\right)
$$

which is consistent with our result at the beginning of this chapter.

\subsection{Trial ansatz}

We make the ansatz

$$
f(\mathbf{k}, g, E, T)=\theta\left(k_{x}\right) \exp \left(-\int_{0}^{k_{x}} \frac{\gamma\left(k_{x}^{\prime}, k_{y}, g, E, T\right)}{e E} d k_{x}^{\prime}-\frac{\pi \hbar v k_{y}^{2}}{e E}\right) .
$$

Inserting the ansatz into the Boltzmann equation yields

$$
\frac{e}{\hbar} E \frac{\partial}{\partial k_{x}} f(\mathbf{k})=\delta\left(\frac{\hbar k_{x}}{e E}\right) \exp \left(-\frac{\pi \hbar v k_{y}^{2}}{e E}\right)-\frac{\gamma(\mathbf{k})}{\hbar} f(\mathbf{k})
$$


which umplies

$$
\gamma(\mathbf{k})=\hbar \frac{I(\mathbf{k} ;[f])}{f(\mathbf{k})}
$$

which demonstrates that $\gamma(\mathbf{k})$ plays the role of a scattering rate, as it occurs in the relaxation time approximation.

It follows either by direct inspection or by using the scaling laws of $I(\mathbf{k} ;[f])$ and $f(\mathbf{k})$ that

$$
\gamma(\mathbf{k}, g, E, T)=b^{-1} \gamma\left(b \mathbf{k}, b^{-1 / 2} g, b^{2} E, b T\right)
$$

If $\gamma=D k$, with $D$ independent on $k$, as it occurs from the relaxation time approximation, it follows

$$
D(g, E, T)=D\left(b^{-1 / 2} g, b^{2} E, b T\right)
$$

At $T=0$ holds that

$$
D(g, E)=D\left(g E^{1 / 4}\right)
$$

To leading order in $g$ we expect that $D$ is quadratic, so we get $D(g, E)=d_{0} g^{2} \sqrt{e E}$ with unknown constant $d_{0}$. A solution based on the above ansatz and consistent with the scaling laws would then be

$$
f(\mathbf{k}, g, E, T)=\theta\left(k_{x}\right) \exp \left(-\frac{d_{0} g^{2}}{\sqrt{e E}} \int_{0}^{k_{x}} k d k_{x}^{\prime}-\frac{\pi \hbar v k_{y}^{2}}{e E}\right) .
$$

It holds

$$
\begin{aligned}
\int_{0}^{k_{x}} k d k_{x}^{\prime} & =\int_{0}^{k_{x}} \sqrt{k_{x}^{\prime 2}+k_{y}^{2}} d k_{x}^{\prime}=k_{y}^{2} \int_{0}^{k_{x} /\left|k_{y}\right|} \sqrt{1+t^{2}} d t \\
& =\frac{k_{y}^{2}}{2}\left(\frac{k_{x}}{\left|k_{y}\right|} \sqrt{1+\left(\frac{k_{x}}{\left|k_{y}\right|}\right)^{2}}+\operatorname{arcsinh}\left(\frac{k_{x}}{\left|k_{y}\right|}\right)\right) \\
& =\left\{\begin{array}{cc}
k_{x}\left|k_{y}\right| & k_{x} \ll\left|k_{y}\right| \\
\frac{1}{2} k_{x}^{2} & k_{x} \gg\left|k_{y}\right|
\end{array}\right. \\
& \simeq k_{x}\left(\left|k_{y}\right|+\frac{1}{2} k_{x}\right)
\end{aligned}
$$

where the last approximation is rather convenient for explicit calculations and does not change any of our fundamental conclusions. It then follows

$$
f(\mathbf{k}, g, E, T)=\theta\left(k_{x}\right) \exp \left(-\frac{d_{0} g^{2}}{\sqrt{e E}} k_{x}\left(\left|k_{y}\right|+\frac{1}{2} k_{x}\right)-\frac{\pi \hbar v k_{y}^{2}}{e E}\right) .
$$


Now we only need to determine the constant $d_{0}$ that is only allowed to depend on $c, v$, and $\hbar$ which are the only remaining variables of the problem. Thus, for $\hbar=1$ the dimension of $d_{0}$ must be some power of velocity $=$ length/time. This puts important constraints on the form of $d_{0}$. The dimension of $\gamma$ is energy. Thus, the dimension of $D$ is energy $\times$ length and it follows indeed that the unit of $d_{0}$ is $\hbar^{-1 / 2}$ velocity $^{-3 / 2}$. Thus, it must hold that

$$
d_{0}=\hbar^{-1 / 2} v^{-3 / 2} \xi\left(\frac{c}{v}\right)
$$

where $\xi(\varepsilon)$ is a dimensionless function with dimensionless argument $\varepsilon=c / v$. It follows

$$
\begin{aligned}
j & =N 2 e v \int \frac{d^{2} k}{(2 \pi)^{2}} \widehat{k}_{x} f(\mathbf{k}) \\
& =N \frac{4 e v}{2 \pi} \int_{-\pi / 2}^{\pi / 2} \frac{d \varphi}{2 \pi} \int_{0}^{\infty} k d k \cos (\varphi) \exp \left(-\left(\frac{d_{0} g^{2} \cos \varphi\left(|\sin \varphi|+\frac{1}{2} \cos \varphi\right)}{\sqrt{e E}}-\frac{\pi \hbar v \sin ^{2} \varphi}{e E}\right) k^{2}\right) \\
& =N \frac{4 e v}{2 \pi} \frac{\sqrt{e E}}{d_{0} g^{2}} \int_{-\pi / 2}^{\pi / 2} \frac{d \varphi}{2 \pi} \frac{\cos (\varphi)}{\cos \varphi\left(|\sin \varphi|+\frac{1}{2} \cos \varphi\right)-\frac{\pi \hbar v}{\sqrt{e E} d_{0} g^{2}} \sin ^{2} \varphi}
\end{aligned}
$$

For large $E$, when

$$
e E \gg\left(\frac{\pi \hbar v}{d_{0} g^{2}}\right)^{2}
$$

we find

$$
j=N \frac{4 e v}{(2 \pi)^{2}} c_{1} \frac{\sqrt{E}}{d_{0} g^{2}}
$$

where

$$
\begin{aligned}
a_{1} & =\int_{-\pi / 2}^{\pi / 2} d \varphi \frac{1}{|\sin \varphi|+\frac{1}{2} \cos \varphi} \\
& =8 \frac{\arctan \left(\frac{2}{\sqrt{5}}\right)-\arctan \left(\frac{1}{\sqrt{5}}\right)}{\sqrt{5}} \simeq 3.443
\end{aligned}
$$

This result for the current helps further to determine $d_{0}$. We know that $j$ diverges if $c / v \rightarrow 0$ since phonons behave as elastic scatteres and no stationary state exitst, i.e. the current diverges in the long time limit. Thus $\xi(\varepsilon \rightarrow 0) \rightarrow 0$. To leading order we expect a $\xi(\varepsilon)=\xi_{0} \varepsilon^{m}$ with some positive exponent $m$. The avalysis of the collision integral shows $m=2$ and we obtain

$$
d_{0}=\hbar^{-1 / 2} \xi_{0} \frac{c^{2}}{v^{7 / 2}}
$$

with dimensionless numerical constant $\xi_{0}$. 


\subsubsection{General dispersion}

To demonstrate that the above assumtion for $\gamma$ is sensible we use the more general form $\gamma=D k^{\nu}$, with $D$ independent on $k$. It follows

$$
D(g, E, T)=b^{\nu-1} D\left(b^{-1 / 2} g, b^{2} E, b T\right) .
$$

At $T=0$ holds that

$$
D(g, E)=\left(\frac{E_{0}}{E}\right)^{\frac{\nu-1}{2}} D\left(g E^{1 / 4}\right) .
$$

To leading order in $g$ we expect that $D$ is quadratic, so we get

$$
D(g, E)=(e E)^{\frac{2-\nu}{2}} d_{0} g^{2}
$$

with unknown constant $d_{0}$. A solution based on the above ansatz and consistent with the scaling laws would then be

$$
f(\mathbf{k}, g, E, T)=\theta\left(k_{x}\right) \exp \left(-\frac{d_{0} g^{2}}{(e E)^{\nu / 2}} \int_{0}^{k_{x}} k^{\nu} d k_{x}^{\prime}-\frac{\pi \hbar v k_{y}^{2}}{e E}\right) .
$$

It holds

$$
\int_{0}^{k_{x}} k^{\nu} d k_{x}^{\prime}=k_{x}\left(\left|k_{y}\right|^{\nu}+\frac{1}{1+\nu} k_{x}^{\nu}\right)
$$

It then follows

$$
f(\mathbf{k}, g, E, T)=\theta\left(k_{x}\right) \exp \left(-\frac{d_{0} g^{2}}{(e E)^{\nu / 2}} k_{x}\left(\left|k_{y}\right|^{\nu}+\frac{1}{1+\nu} k_{x}^{\nu}\right)-\frac{\pi \hbar v k_{y}^{2}}{e E}\right) .
$$

It follows

$$
\begin{aligned}
j & =N 2 e v \int \frac{d^{2} k}{(2 \pi)^{2}} \widehat{k}_{x} f(\mathbf{k}) \\
& =N \frac{4 e v}{2 \pi} \int_{-\pi / 2}^{\pi / 2} \frac{d \varphi}{2 \pi} \int_{0}^{\infty} k d k \cos (\varphi) e^{-\frac{d_{0} g^{2}}{(e E)^{\nu / 2}} \cos \varphi\left(|\sin \varphi|^{\nu}+\frac{\cos \varphi}{1+\nu}\right) k^{\nu+1}-\frac{\pi \hbar v \sin ^{2} \varphi}{e E} k^{2}}
\end{aligned}
$$

It holds

$$
\int_{0}^{\infty} k d k e^{-\frac{a}{(e E)^{\nu / 2}} k^{\nu+1}-\frac{b}{e E} k^{2}}=\frac{(e E)^{\frac{\nu}{\nu+1}}}{a^{\frac{2}{\nu+1}}} \int_{0}^{\infty} x d x e^{-x^{\nu+1}-b\left(a^{2} e E\right)^{-\frac{1}{\nu+1}} x^{2}}
$$

For sufficiently large $E$, we can ignore the second term in the exponent and obtain that

$$
j \propto g^{-\frac{4}{\nu+1}}(e E)^{\frac{\nu}{\nu+1}}
$$

This only reproduces the requires scaling behavior $j \propto g^{-2} \sqrt{e E}$ if $\nu=1$. Thus, it must hold that the scattering rate is linear in momentum. 


\subsubsection{Energy density}

The energy density of the system is

$$
\mathcal{E}=N \int \frac{d^{2} k}{(2 \pi)^{2}} \sum_{\lambda} \varepsilon_{\mathbf{k} \lambda} f_{\mathbf{k} \lambda}
$$

where $N=4$ reflects the valley and spin dedegeneracy. It follows from the Boltzmann equation that the change in energy $Q=d \mathcal{E} / d t$ is given as

$$
Q=N \int \frac{d^{2} k}{(2 \pi)^{2}} \sum_{\lambda} \varepsilon_{\mathbf{k} \lambda}\left(I_{\lambda}(\mathbf{k})+S_{\lambda}(\mathbf{k})\right) .
$$

The unit of $Q$ is $\frac{\text { energy }}{\text { length }^{2} \times \text { time }}$ For a stationary state one expects that $Q=0$. The first term is the cooling power due to the coupling to the heat bath, while the second term is the Joule heating due to the pair production. It holds for the latter

$$
Q_{s}=N \int \frac{d^{2} k}{(2 \pi)^{2}} \sum_{\lambda} \varepsilon_{\mathbf{k} \lambda} S_{\lambda}(\mathbf{k})=\frac{N}{(2 \pi)^{2}} \frac{2 e^{2}}{\pi \hbar} E^{2}
$$

In this analysis we used the Schwinger term as discussed above. However, the Schwinger source term was derived for noninteracting electrons and in an interacting problem one expects a backreaction that leads to a modification of the source term taking into account pair creation when there are particles present in the initial state. This yields [Kluger et al. (1998)]

$$
S_{\lambda}(\mathbf{k})=\left(f_{\mathbf{k} \bar{\lambda}}-f_{\mathbf{k} \lambda}\right) S_{\lambda}^{\mathrm{vac}}(\mathbf{k})
$$

In case of $\lambda=+$ follows at particle hole symmetry:

$$
S(\mathbf{k})=\left(1-2 f_{\mathbf{k}}\right) S^{\mathrm{vac}}(\mathbf{k})
$$

Inserting this into the $Q_{s}$ yields $Q_{s} \propto k_{\text {typ }}^{2} E$, where $k_{\text {typ }}^{2}$ is the typical momentum of the system that for $T=0$ must be of the form

$$
k_{\text {typ }}^{2}=E \Theta\left(g E^{1 / 4}\right)
$$

From our trial form for the distribution function we find $k_{\text {typ }}^{2} \simeq \sqrt{e E} /\left(d_{0} g^{2}\right)$ which corresponds to $\Theta(x) \propto x^{-2}$. Keeping in mind that $j=N 2 e v \int \frac{d^{2} k}{(2 \pi)^{2}} \widehat{k}_{x} f(\mathbf{k})$ implies that $j \simeq N e v k_{\text {typ }}^{2} \simeq$ $N e v \sqrt{e E} /\left(d_{0} g^{2}\right)$, we see that including back-reaction terms

$$
Q_{s}=j E
$$


as expected from Joule heating. Inserting $k_{\text {typ }}^{2}$ yields

$$
Q_{s}=N v(e E)^{3 / 2} /\left(d_{0} g^{2}\right)
$$

To analyse the relaxational part we write

$$
\begin{aligned}
Q_{r e l}(g, E, T) & =2 N \int \frac{d^{2} k}{(2 \pi)^{2}} \varepsilon_{\mathbf{k}} I(\mathbf{k}, g, T ;[f]) \\
& =2 N \int \frac{d^{2} k}{(2 \pi)^{2}} \varepsilon_{\mathbf{k}} \frac{1}{b} I\left(b \mathbf{k}, b^{-1 / 2} g, b T ;\left[f^{\prime}\right]\right) \\
& =b^{-4} 2 N \int \frac{d^{2} k}{(2 \pi)^{2}} \varepsilon_{\mathbf{k}} I\left(\mathbf{k}, b^{-1 / 2} g, b T ;\left[f^{\prime}\right]\right) \\
& =b^{-4} Q_{r e l}\left(b^{-1 / 2} g, b^{2} E, b T\right)
\end{aligned}
$$

In case of our trial function we know that $I(\mathbf{k} ;[f])=-\hbar^{-1} \gamma(\mathbf{k}) f(\mathbf{k})$ and we obtain

$$
Q_{r e l}(g, E, T)=-\frac{2 N}{\hbar} \int \frac{d^{2} k}{(2 \pi)^{2}} \varepsilon_{\mathbf{k}} \gamma(\mathbf{k}) f(\mathbf{k})
$$

We can first estimate this expression qualitatively and find

$$
\begin{aligned}
Q_{r e l}(g, E, T) & \simeq-N v k_{\mathrm{typ}}^{4} d_{0} g^{2} \sqrt{e E} \\
& \simeq-N v(e E)^{3 / 2} /\left(d_{0} g^{2}\right)
\end{aligned}
$$

The fact that $Q_{s} \simeq-Q_{\text {rel }}$ demonstrates that the trial solution is indeed an acceptable stationary solution. The notion of a typical momentum scale in the distribution function can also be used to introduce an effective electron temperature

$$
k_{B} T_{*}=\hbar v k_{\mathrm{typ}}
$$

which yields

$$
k_{B} T_{*}=\frac{\hbar v(e E)^{1 / 4}}{d_{0}^{1 / 2} g}
$$

This can also be seen if one explicitly determines the effective temperature via

$$
\begin{aligned}
\left(k_{B} T^{*}\right)^{2} & \simeq(\hbar v)^{2} \int_{0}^{\infty} k d k \int_{0}^{2 \pi} d \varphi k\left(-\frac{\partial f}{\partial k}\right) \\
& =(\hbar v)^{2} \frac{\sqrt{e E}}{d_{0} g^{2}} \int_{0}^{\pi / 2} d \varphi \frac{1}{\cos \varphi\left(|\sin \varphi|+\frac{1}{2} \cos \varphi\right)^{2}}
\end{aligned}
$$




\section{CHAPTER 5. Relaxation time approximation}

In this chapter, we want to see how the current reacts to a large electric field. Tf we assume a simplified form of the collision operator with fixed, momentum independent relaxation rate. We will then solve this simplified problem using two distinct approaches: i) we will directly solve the Boltzmann equation and ii) we will use the scaling approach used in the previous approach. The fact that both methods yield the same result

$$
J \propto E^{\frac{3}{2}} \tau
$$

is further evidence for the reliability of the scaling approach.

\subsection{Explicit Result for Relaxation Time Approximation}

To proceed we use dimensionless momentum variables

$$
\mathbf{p}=\beta \hbar v \mathbf{k}
$$

and use $\nabla_{\mathbf{k}}=\hbar \beta v \nabla_{\mathbf{p}}$ such that the Boltzmann equation becomes with $\mathbf{F}=e \tau \beta v \mathbf{E}$

$$
\mathbf{F} \cdot \nabla_{\mathbf{p}} f_{\mathbf{p} \lambda}=-f_{\mathbf{p} \lambda}+f_{p \lambda}^{e q}+\lambda \delta\left(p_{x} / F\right) \exp \left(-\frac{\pi p_{y}^{2} k_{B} T}{F \hbar \tau^{-1}}\right)
$$

where $f_{p \lambda}^{e q}=\frac{1}{e^{\lambda p}+1}$. This yields for the current

$$
\mathbf{j}=\frac{e T^{2}}{v} \int_{\mathbf{p}} \sum_{\lambda= \pm} \lambda \widehat{\mathbf{p}} f_{\mathbf{p} \lambda}
$$

We want to solve the equations via Fourier transform. Note that $f_{+}(\mathbf{p} \rightarrow \infty) \rightarrow 0$ while $f_{-}(\mathbf{p} \rightarrow \infty) \rightarrow 1$. Thus, we rather consider $f_{h}(\mathbf{p})=1-f_{-}(\mathbf{p})$. In analogy we call $f_{e}(\mathbf{p})=$ $f_{+}(\mathbf{p})$. At the Dirac point, $f_{e}(\mathbf{p})$ and $f_{h}(\mathbf{p})$ obey the same equation

$$
F \partial_{p_{x}} f(\mathbf{p})=-f(\mathbf{p})+f^{e q}(p)+\delta\left(p_{x} / F\right) \exp \left(-\frac{\pi p_{y}^{2} k_{B} T}{F \hbar \tau^{-1}}\right)
$$


with same boundary conditions. For the current follows then

$$
\mathbf{j}=\frac{2 e T^{2}}{v \hbar^{2}} \int \frac{d^{2} p}{(2 \pi)^{2}} \widehat{\mathbf{p}} f(\mathbf{p}),
$$

where we used that $\int d^{2} p \widehat{\mathbf{p}}=0$.

Applying Fourier transformation to 5.4 to eliminate the derivative and then applying Fourier transformation again, we obtain the distribution function $f(\mathbf{p})$ whose contributions come from two terms: relaxation term $f^{r e l}(\mathbf{p})$ and Schwinger pair-production term $f^{s}(\mathbf{p})$,

$$
f(\mathbf{p})=f^{r e l}(\mathbf{p})+f^{s}(\mathbf{p})
$$

where

$$
\begin{aligned}
f^{r e l}(\mathbf{p}) & =\frac{1}{|F|} \int_{-\infty}^{\infty} d p_{x}^{\prime} \frac{1}{e^{p^{\prime}}+1} e^{-\frac{\left|p_{x}-p_{x}^{\prime}\right|}{|F|}} \Theta\left(\frac{p_{x}-p_{x}^{\prime}}{F}\right) \\
f^{s}(\mathbf{p}) & =\operatorname{sign}(F) \exp \left(-\frac{\pi p_{y}^{2} k_{B} T}{F \hbar \tau^{-1}}\right) e^{-\frac{\left|p_{x}\right|}{|F|}} \Theta\left(\frac{p_{x}}{F}\right)
\end{aligned}
$$

Lets consider without restriction that $F>0$. Then it follows for the distribution function that:

$$
\begin{aligned}
f^{r e l}(\mathbf{p}) & =\int_{0}^{\infty} d s \frac{e^{-s}}{\exp \left(\sqrt{\left(p_{x}-F s\right)^{2}+p_{y}^{2}}\right)+1} \\
f^{s}(\mathbf{p}) & =\Theta\left(p_{x}\right) \exp \left(-\frac{\pi p_{y}^{2} \tau k_{B} T / \hbar+p_{x}}{F}\right) . \\
& =\Theta\left(p_{x}\right) \exp \left(-\frac{v \pi k_{y}^{2}+\tau^{-1} k_{x}}{e E / \hbar}\right)
\end{aligned}
$$

The expression for $f^{r e l}(\mathbf{p})$ is physically insightful if one returns to original units

$$
f^{r e l}(\mathbf{k})=\int_{0}^{\infty} d t \frac{\tau^{-1} e^{-t / \tau}}{\exp \left(\beta v \hbar \sqrt{\left(k_{x}-e E t\right)^{2}+k_{y}^{2}}\right)+1}=\int_{0}^{\infty} \frac{d t}{\tau} e^{-t / \tau} f^{e q}(v|\mathbf{K}(t)|)
$$

where now $f^{e q}(\varepsilon)=1 /\left(e^{\beta \varepsilon}+1\right)$ and $\mathbf{K}(t)=\mathbf{k}-e \mathbf{A}(t)$.

We can now determine the current. For the analysis of $j_{x}^{r e l}$ we can now determine the current (returning to dimensionless units)

$$
\mathbf{j}=\frac{2 e T^{2}}{v} \int_{0}^{\infty} d s e^{-s} \int \frac{d^{2} p}{(2 \pi)^{2}} \widehat{\mathbf{p}} f^{e q}(|\mathbf{P}(t)|)
$$


If we again consider a field along the $x$-direction

$$
\begin{aligned}
j_{x} & =\frac{2 e T^{2}}{v} \int_{0}^{\infty} d s e^{-s} \int \frac{d^{2} p}{(2 \pi)^{2}} \frac{p_{x}}{\sqrt{p_{x}^{2}+p_{y}^{2}}} \frac{1}{\exp \left(\sqrt{\left(p_{x}-F s\right)^{2}+p_{y}^{2}}\right)+1} \\
& =\frac{2 e T^{2}}{v} \int_{0}^{\infty} d s e^{-s} \int \frac{d^{2} p}{(2 \pi)^{2}} \frac{\cos \varphi+F s / p}{\sqrt{(\cos \varphi+F s / p)^{2}+\sin ^{2} \varphi}} \frac{1}{e^{p}+1}
\end{aligned}
$$

We substitute $u=s / p$ and obtain

$$
\begin{aligned}
j_{x} & =\frac{2 e T^{2}}{v} \int \frac{p d p d \varphi}{(2 \pi)^{2}} \int_{0}^{\infty} d u \frac{\cos \varphi+F u}{\sqrt{(\cos \varphi+F u)^{2}+\sin ^{2} \varphi}} \frac{p e^{-u p}}{e^{p}+1} \\
& =\frac{e T^{2}}{v \pi} \int_{0}^{\infty} d u L(F u) G(u),
\end{aligned}
$$

where we introduced

$$
\begin{aligned}
G(u) & =\int_{0}^{\infty} d p \frac{p^{2} e^{-u p}}{e^{p}+1} \\
& =\frac{1}{4}\left(\zeta\left(3, \frac{1+u}{2}\right)-\zeta\left(3, \frac{2+u}{2}\right)\right)
\end{aligned}
$$

and

$$
L(a)=\int_{0}^{2 \pi} \frac{d \varphi}{2 \pi} \frac{\cos \varphi+a}{\sqrt{(\cos \varphi+a)^{2}+\sin ^{2} \varphi}} .
$$

$L$ (a) can be expressed in terms of elliptic integrals. It holds

$$
G(u)=\left\{\begin{array}{cc}
\frac{3 \zeta(3)}{2}-\frac{7 \pi^{4}}{120} u & u \ll 1 \\
u^{-3} & u \gg 1
\end{array}\right.
$$

while

$$
L(a)=\left\{\begin{array}{cc}
\frac{a}{2}+\frac{a^{3}}{16} & a \ll 1 \\
1-\frac{1}{4} a^{-2} & a \gg 1
\end{array}\right.
$$

At large field we have $L(F u) \rightarrow 1$ and we find that the current saturates at

$$
j_{x}(F \rightarrow \infty)=\frac{\pi e T^{2}}{12 v}=\frac{\pi}{12} \frac{e\left(k_{B} T\right)^{2}}{v \hbar^{2}}
$$

Next we analyze the current contribution due to the Schwinger mechanism.

$$
\begin{aligned}
j_{x}^{s}= & \frac{2 e T^{2}}{v \hbar^{2}} \int \frac{d^{2} p}{(2 \pi)^{2}} \widehat{p}_{x} \theta\left(p_{x}\right) \exp \left(-\frac{\pi p_{y}^{2} \tau k_{B} T / \hbar+p_{x}}{F}\right) \\
& =\frac{e T^{2}}{2 \pi^{2} \hbar^{2} v} \int_{-\infty}^{\infty} d p_{y} \int_{0}^{\infty} d p_{x} \frac{p_{x}}{\sqrt{p_{x}^{2}+p_{y}^{2}}} \exp \left(-\frac{\pi p_{y}^{2} \tau k_{B} T / \hbar+p_{x}}{F}\right)
\end{aligned}
$$


We first perform the integral with respect to $p_{y}$ and it follows

$$
\begin{aligned}
j_{x}^{s} & =\frac{e k_{B}^{2} T^{2}}{2 \pi^{2} \hbar^{2} v} \int_{0}^{\infty} d p_{x} p_{x} \exp \left(-\frac{p_{x}}{F}+\frac{\pi p_{x}^{2} \tau k_{B} T / \hbar}{2 F}\right) K_{0}\left(\frac{\pi p_{x}^{2} \tau k_{B} T / \hbar}{2 F}\right) \\
& =\frac{e k_{B} T \tau^{-1} F}{\hbar 2 \pi^{3} v} \int_{0}^{\infty} d u \exp \left(-\sqrt{\frac{u}{u_{0}}}+u\right) K_{0}(u)
\end{aligned}
$$

where

$$
u_{0}=\frac{\pi k_{B} T}{2 \hbar \tau^{-1}} F
$$

In the original units this becomes

$$
j^{s}=\frac{e^{2} E}{h \pi^{2}} \int_{0}^{\infty} d u \exp \left(-\sqrt{\frac{u}{u_{0}}}+u\right) K_{0}(u)
$$

with

$$
u_{0}=\frac{\pi \tau^{2} v}{2 \hbar} e E
$$

At high field we need to analyze large $u_{0}$. Now the integral is dominated by the large $u$ behavior of $\exp (u) K_{0}(u) \simeq \sqrt{\frac{\pi}{2 u}}$. It holds

$$
\int_{0}^{\infty} d u e^{-\sqrt{\frac{u}{u_{0}}}} \sqrt{\frac{\pi}{2 u}}=2 u_{0}^{1 / 2}
$$

Such that

$$
j^{s}=\frac{e^{2} E^{3 / 2} \tau}{h \pi^{2}} \sqrt{2 \pi v e / \hbar}
$$

Due to the $E^{\frac{3}{2}}$ dependence, current contributed from pair-production easily outrivals the current from scattering process. This result is consistent with the result from section 3.2, but now there

is a restriction on the time which results in the finite scattering rate $\frac{1}{\tau}$. After such a laborious work, we will show how to obtain the same conclusion for electric field and scattering rate dependence in a simpler way based on scaling argument.

\subsection{Scaling argument for relaxation time approximation}

For Boltzmann equation in relaxation times approximation, the collision integral $I_{\lambda}$ can be written as written as

$$
I_{\lambda}=-\frac{f_{\mathbf{k} \lambda}-f_{\mathbf{k} \lambda}^{e q}}{\tau_{p h}}
$$


with $\tau_{p h}^{-1}$ acting as scattering rate. For a clean graphene with $\tau_{p h}$ a constant and the equilibrium distribution function is

$$
f_{\mathbf{k} \lambda}^{e q}=\frac{1}{e^{\lambda \beta v k \hbar}+1}
$$

7

Here we use scaling to exam the current behavior at large field limit in relaxation time approximation. From the previous chapter, we know that $e \mathbf{E} \cdot \nabla_{\mathbf{k}} f$ scales as $\frac{1}{b}$. Therefore the collision integral must scale as $\frac{1}{b}$ as well. From

$$
\tau_{p h}=b^{\gamma} \tau_{p h}^{\prime}
$$

we obtain the scaling law of collision integral:

$$
\frac{f_{\mathbf{k} \lambda}-f_{\mathbf{k} \lambda}^{e q}}{\tau_{p h}}=\frac{f_{\mathbf{k} \lambda}-f_{\mathbf{k} \lambda}^{e q}}{b^{-\gamma} \tau_{p h}^{\prime}}=\frac{f_{\mathbf{k} \lambda}-f_{\mathbf{k} \lambda}^{e q}}{b \tau_{p h}}
$$

so we know that $\gamma=-1$. In this part, $\tau_{p h}^{-1}$ takes the place of coupling constant $g$ in the previous chapter. Here the current can be written as

$$
\begin{aligned}
J_{r e l} & =\sum_{\lambda= \pm} \int k d k \cos \theta d \theta f_{\lambda}\left(k_{x}, k_{y}, T, E, \tau\right) \\
& =\frac{1}{b^{2}} \sum_{\lambda= \pm} \int k^{\prime} d k^{\prime} \cos \theta d \theta f_{\lambda}\left(k_{x}^{\prime}, k_{y}^{\prime}, b T, b^{2} E, b^{-1} \tau\right) \\
& =\frac{1}{b^{2}} \Psi\left(b T, b^{2} E, b^{-1} \tau\right)
\end{aligned}
$$

Again we choose $b^{2} E=E_{0}$ and $T=0$ as nonlinear transport limit.

$$
J_{r e l}=E \Psi(\sqrt{E} \tau)
$$

In the classical model, like Drude model, the conductivity is proportional to the relaxation rate $\tau$. From the relation between $J$ and $\Psi$ shown above, we know that $\Psi$ represents the conductivity, therefore it must obey

$$
\Psi(x) \propto x
$$

so we obtain the nonlinear current in relaxation time approximation as

$$
J_{\text {rel }} \propto E^{\frac{3}{2}} \tau
$$


which is a very different result $J \propto E^{\frac{1}{2}}$ as shown in the previous chapter. There is no contradiction between these two results. The only reason we have 5.32 is because we assume that scattering rate is a constant while in the realistic situation it is not a constant. So the correct answer for nonlinear current is $J \propto E^{\frac{1}{2}}$. The consistency between 5.32 and 5.24 provides us with a confidence that scaling is a useful tool which gives us the correct transport behavior in nonlinear regime without solving the nonlinear integro-differential Boltzmann equation. Of course our ultimate goal is obtaining the distribution function, both Boltzmann transport and scaling serve as powerful tools making us unveil the mysterious distribution function. 


\section{CHAPTER 6. Summary}

In this thesis, we have shown that using both scaling argument and Boltzmann transport theory enable us understanding nonlinear electric transport behavior and the key features of distribution function. To summarize our results, we know that the electric currrent behaves as

$$
J \propto \frac{\sqrt{E}}{g^{2}}
$$

at large field under the assumption that conductivity is still proportion to $\frac{1}{g^{2}}$ in nonlinear regime. Here $\mathrm{g}$ is the coupling constant between electrons and acoustic phonons. Second, based on the result shown above and the ansatz for distribution function, we know that phonon-induced scattering is more important than the electron-electron spontaneous decay and the resulting energy balance gives an effective temperature

$$
T^{*} \propto \frac{E^{\frac{1}{2}}}{g}
$$

which is a nontrivial result compared with the result done by other scientists [Viljas and Heikkila (2010)]. In the end, we use the relaxation time approximation as an example of showing the reliability of scaling approach by comparing the results done by explicit calculation and scaling.

These results are clearly only the first steps towards a more complete understanding of the nonequilibrium transport of graphene. First, a complete numerical solution of the nonlinear Boltzmann equation is needed to confirm the scaling theory and to be the starting point for further examinations. Second in our investigation we have assumed that the heat produced by large electric fields is instantly transported to the substrate which acts as a heat bath. A more detailed analysis of these heat transport processes are important in order to demonstrate that this is indeed a realistic scenario. Finally, to investigate free standing graphene layers, we need to analyze finite samples and investigate the electron and phonon heat conductivity. In this case 
we expect that the heat transport is dominated by electron heat conduction. This brings us back to the necessity to investigate electron-electron and electron-phonon scattering processes in one combined hot electron transport theory, which is the intellectually most interesting and challenging part of our future investigation. The results obtained here are a necessary prerequisites for these future investigations. 


\section{BIBLIOGRAPHY}

Arai, M. R. (1983). A fundamental noise limit for biased resistors at low temperatures. Appl. Phys. Lett., 42:906. 17

Ashcroft, N. W. and Mermin, N. D. (1976). Solid State Physics. Holt, Rinehart and Winston, New York, 1976. 18

Beenakker, C. W. J. (2008). Colloquium: Andreev reflection and klein tunneling in graphene. Rev. Mod. Phys, 80:1337. 1

Cheianov, V. V. and Fal'ko, V. I. (2006). Selective transmission of dirac electrons and ballistic magnetoresistance of n-p junctions in graphene. Phys. Rev. B, 41:041403(R). 1

Dora, B. and Moessner, R. (2010). Nonlinear electric transport in graphene: Quantum quench dynamics and the schwinger mechansim. Phys. Rev. B, 81:165431. 1, 19

Fritz, L., Schmalian, J., Muller, M., and Sachdev, S. (2008). Quantum critical transport in clean graphene. Phys. Rev B, 78:085416. 1

Green, A. G. and Sondhi, S. L. (2005). Nonlinear quantum critical transport and the schwinger mechanism or a superfluid-mott-insulator transition of bosons. Phy. Rev. Lett., 95:267001. 22

Grosso, G. and Parravicini, G. P. (2000). Solid State Physics. Academic Press. 14

Hentschel, M. and Guinea, F. (2007). Orthogonality catastrophe and kondo effect in graphene. Phys. Rev B, 76:115404. 1 
Hwang, E. H. and Sarma, S. D. (2008). Acoustic phonon scattering limited carrier mobility in two-dimensional extrinsic graphene. Phys. Rev. B, 77:115449. 1, 28, 29

Katsnelson, M. I. (2006). Zitterbewegung, chirality, and minimal conductivity in graphene. Eur. Phys. J. B, 51:157. 1

Kluger, Y., Mottola, E., and Eisenberg, J. M. (1998). Quantum vlasov equation and its markov limit. Phys. Rev D, 58:125015. 35

Kusminskiy, S. V., Campbell, D. K., and Neto, A. H. C. (2009). Lenosky's energy and the phonon dispersion of graphene. Phys. Rev B, 80:035401. 2

Lewkowicz, M. and Rosenstein, B. (2009). Dynamics of particle-hole pair creation in graphene. Phy. Rev. Lett., 102:106802. 22

Mishchenko, E. G. (2009). Dynamic conductivity in graphene beyond linear rsponse. Phys. Rev. Lett, 103:246802. 1

Neto, A. H. C., Guinea, F., Peres, N. M. R., Novoselov, K. S., and Geim, A. K. (2009). The electronic properties of graphene. Rev. Mod. Phys., 81:109. 1

Novoselov, K. S., Geim, A. K., Morozov, S. V., Jiang, D., Katsnelson, M. I., Grigorieva, I. V., Dubonos, S. V., and Firsov, A. A. (2005). Two-dimensional gas of massless dirac fermions in graphene. Nature (London), 438:197. 1

Novoselov, K. S., Geim, A. K., Morozov, S. V., Jiang, D., Zhang, Y., Dubonos, S. V., Grigorieva, I. V., and Firsov, A. A. (2004). Electric field effect in atomically thin carbon films. Science, 306:666. 1

Petroski, H. (1989). The Pencil: A History of Design and Circumstance. Knopf. 1

Prange, R. E. and Kadanoff, L. P. (1964). Transport theory for electron-phonon interaction in metals. Phy. Rev, 134:A566. 24

Satou, A., Vasko, F. T., and Ryzhii, V. (2008). Nonequilibrium carriers in intrinsic graphene under interband photoexcitation. Phys. Rev. B, 78:115431. 1 
Schwinger, J. (1951). On gauge invariance and vacuum polarization. Phys. Rev, 82:664. 1, 22

Stauber, T., Peres, N. M., and Guinea, F. (2007). Electronic transport in graphene: A semiclassical approach including midgap states. Phys. Rev B, 76:205423. 29

Tanji, N. (2009). Dynamical view of pair creation in uniform electric and magnetic fields. Ann. Phys., 324:1691. 22

Vasko, F. T. and Ryzhii, V. (2008). Photoconductivity of intrinsic graphene. Phys. Rev. B, $77: 195433.1$

Viljas, J. K. and Heikkila, T. T. (2010). Electron-phonon heat transfer in monolayer and bilayer graphene. Phys. Rev B, 81:245404. 43

Yan, J., Zhang, Y., Kim, P., and Pinczuk, A. (2007). Electric field effect tuning of electronphonon coupling in graphene. Phys. Rev. Lett, 98:166802. 1

Zener, C. (1932). Non-adiabatic crossing of energy levels. Proc. R. Soc, London, Ser:A, 696:1932. 14 